\title{
Revision of optical klystron enhancement effects in self-amplified spontaneous emission free electron lasers
}

\author{
Gianluca Geloni, ${ }^{1, *}$ Marc Guetg $\odot,{ }^{2}$ Svitozar Serkez $\odot,{ }^{1}$ and Evgeny Schneidmiller $\circledast^{2}$ \\ ${ }^{1}$ European XFEL, Holzkoppel 4, 22869 Schenefeld, Germany \\ ${ }^{2}$ Deutsches Electronen Synchrotron, Notkestrasse 85, 22867 Hamburg, Germany
}

(Received 12 April 2021; accepted 9 September 2021; published 28 September 2021)

\begin{abstract}
We review the theory of optical klystrons, with its applications for self-amplified spontaneous emission free electron lasers in mind. We show that previous theories miss terms in the power gain factor that cannot be neglected, and we illustrate differences between the previously known analytical expressions, new ones found in this paper, and numerical calculations. We then consider the use of optical klystrons for electron energy-spread and radiation coherence-time diagnostics purposes.
\end{abstract}

DOI: 10.1103/PhysRevAccelBeams.24.090702

\section{INTRODUCTION}

A free electron laser (FEL)-based optical klystron [1-8] is a device constituted by two FEL radiators with a longitudinal dispersion element in between. Figure 1 sketches a typical realization in the case of a segmented FEL with tunable undulator gaps, where an undulator segment is removed and substituted by a magnetic chicane, and the longitudinal dispersion can be finely tuned with the help of a phase shifter belonging to the original setup. Similar realizations are currently available at all major XFEL facilities. To give an example, optical klystron capabilities are currently present both at the SASE2 and SASE3 undulator lines of the European XFEL, where magnetic chicanes are installed as part, respectively, of hard $\mathrm{x}$-ray self-seeding and two-color pump-probe setups, see $[9,10]$. Optical klystrons allow for optimizing the electron bunching process with the help of a nonzero longitudinal dispersion. The final FEL output results are thus enhanced with respect to the usual case, which is recovered when the chicane is switched off. Since the behavior of the power gain factor on the strength of the dispersion depends parametrically on the electron energy spread, the system can be used in order to diagnose this important parameter. It has also been considered [11] to use optical klystron-based measurements to investigate the coherence properties of the FEL pulse directly in the time domain.

The widespread availability of the setup, together with its many uses, makes the theory behind it particularly

\footnotetext{
*gianluca.geloni@xfel.eu
}

Published by the American Physical Society under the terms of the Creative Commons Attribution 4.0 International license. Further distribution of this work must maintain attribution to the author(s) and the published article's title, journal citation, and DOI. important, especially when it is applied to retrieve relevant electron or radiation characteristics. A theory of FEL-based optical klystrons was developed in the FEL community already several years ago [2]. In this paper we revisit it, showing that important terms in the analytical expression for the power gain factor are missing, namely those involving products of the dispersion strength parameter and the energy spread parameter.

In Sec. II B we review the one-dimensional theory, basically confirming the general expression for the optical klystron enhancement factor found in $[2,8]$. Upon expansion for a small energy spread parameter, however, we find deviations from previously reported expressions for the power gain factor. These deviations are ascribed to the fact that, in previous treatments, the dispersion strength parameter was not taken to be of order of the inverse energy spread parameter, and was thus neglected. In Sec. III we compare our analytical expressions with those available in literature and with numerical calculations. We then discuss ways to use the theory to come to a determination of the energy spread of the electron beam and of the coherence time of the radiation pulse. Finally, we come to a discussion about the applicability of the one-dimensional theory and to conclusions.

\section{THEORY}

\section{A. Solution of the initial value problem}

We solve the FEL initial value problem using the Vlasov equation approach in [1] (paragraph 2.14) for the

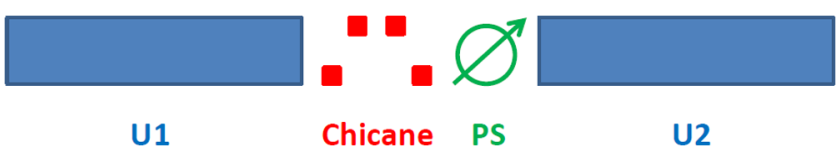

FIG. 1. Sketch of an optical klystron arrangement at a segmented FEL with tunable undulator gaps. 
one-dimensional steady-state FEL problem, slightly generalizing it for a non-negligible energy spread. The coordinates of an electron in the longitudinal phase space are specified by the electron energy deviation from the nominal value $\mathcal{E}_{0}$, which we indicate with $P=\mathcal{E}-\mathcal{E}_{0}$, and by the ponderomotive phase $\psi=k_{u} z+\omega(z / c-t)$ with $k_{u}=2 \pi / \lambda_{u}, \lambda_{u}$ being the undulator period.

We indicate with $\widetilde{E}$ the slowly varying amplitude of the complex electric field at frequency $\omega$. The equation for the evolution of $\tilde{E}$ along the undulator is then

$$
\begin{aligned}
\frac{d \tilde{E}}{d \hat{z}}= & \int_{0}^{\hat{z}} d \hat{z}^{\prime} \tilde{E}\left(\hat{z}^{\prime}\right) \int_{-\infty}^{\infty} d \hat{P} \frac{d \hat{F}}{d \hat{P}} \exp \left[i(\hat{P}+\hat{C})\left(\hat{z}^{\prime}-\hat{z}\right)\right] \\
& +\int_{-\infty}^{\infty} d \hat{P} \hat{f}_{1 \mid \hat{z}=0}(\hat{P}) \exp [-i(\hat{P}+\hat{C}) \hat{z}] .
\end{aligned}
$$

We kept the same notations in [1] and in particular the definition of normalized quantities: $\hat{z}=z \rho \omega /\left(\gamma_{z}^{2} c\right)$, with $z$ the distance traveled inside the undulator, $\rho$ the 1D FEL parameter, $\quad \gamma_{z}=\left[1-\bar{v}_{z}^{2}\left(\mathcal{E}_{0}\right) / c^{2}\right]^{-1 / 2}$ the longitudinal Lorentz factor of an electron with nominal electron energy $\mathcal{E}_{0}$ and average longitudinal velocity $\bar{v}_{z}\left(\mathcal{E}_{0}\right)$, and $c$ the speed of light in vacuum; $\hat{P}=P /\left(\rho \mathcal{E}_{0}\right) ; \hat{C}=C \gamma_{z}^{2} c /(\rho \omega)$, with $C=k_{u}+\omega / c-\omega / \bar{v}_{z}\left(\mathcal{E}_{0}\right)$ the detuning from resonance of the nominal electron; $\hat{f}_{1}=2 \pi e K \rho^{2} \omega \mathcal{E}_{0} /$ $\left(\gamma_{z}^{2} \gamma c\right) \tilde{f}_{1}$, with $K$ the maximum ${ }^{1}$ undulator parameter and $\tilde{f}_{1}$ the slowly varying complex amplitude of the electron density modulation at the fundamental harmonic; $\hat{F}=\rho \mathcal{E}_{0} F$, with $F(P)$ the energy spread distribution.

The solution through the Laplace technique gives

$$
\tilde{E}(\hat{z})=\left[\frac{E_{0}}{1-\hat{\mathcal{D}}_{\mid \lambda=\lambda_{1}}^{\prime}}+\frac{\hat{\mathcal{D}}_{f \mid \lambda=\lambda_{1}}}{1-\hat{\mathcal{D}}_{\mid \lambda=\lambda_{1}}^{\prime}}\right] \exp \left(\lambda_{1} \hat{z}\right)
$$

where $E_{0}$ is an initial external field amplitude and we defined

$$
\hat{\mathcal{D}}= \begin{cases}\int_{-\infty}^{\infty} d \hat{P} \frac{\hat{F}^{\prime}(\hat{P})}{\lambda+i(\hat{P}+\hat{C})} & \operatorname{Re}(\lambda)>0 \\ \text { P.V. } \int_{-\infty}^{\infty} d \hat{P} \frac{\hat{F}^{\prime}(\hat{P})}{\lambda+i(\hat{P}+\hat{C})}+\pi \hat{F}^{\prime}(i \lambda-\hat{C}) & \operatorname{Re}(\lambda)=0 \\ \int_{-\infty}^{\infty} d \hat{P} \frac{\hat{F}^{\prime}(\hat{P})}{\lambda+i(\hat{P}+\hat{C})}+2 \pi \hat{F}^{\prime}(i \lambda-\hat{C}) & \operatorname{Re}(\lambda)<0\end{cases}
$$

and

\footnotetext{
${ }^{1}$ The original derivation in [1] deals with a helical undulator, which we follow here. Generalization to the case of a planar undulator can be obtained by using $K_{\text {rms }}$ and considering a modified coupling factor between radiation and electrons. The final result in terms of enhancement factor does not change.
}

$$
\hat{\mathcal{D}}_{f}= \begin{cases}\int_{-\infty}^{\infty} d \hat{P} \frac{\hat{f}_{1 \mid \hat{z}=0}(\hat{P})}{\lambda+i(\hat{P}+\hat{C})} & \operatorname{Re}(\lambda)>0 \\ \text { P.V. } \int_{-\infty}^{\infty} d \hat{P} \frac{\hat{f}_{1 \mid z=0}(\hat{P})}{\lambda+i(\hat{P}+\hat{C})}+\pi \hat{f}_{1 \mid \hat{z}=0}(i \lambda-\hat{C}) & \operatorname{Re}(\lambda)=0 \\ \int_{-\infty}^{\infty} d \hat{P} \frac{\hat{f}_{1 \mid z=0}(\hat{P})}{\lambda+i(\hat{P}+\hat{C})}+2 \pi \hat{f}_{1 \mid \hat{z}=0}(i \lambda-\hat{C}) & \operatorname{Re}(\lambda)<0,\end{cases}
$$

P.V. indicating the principal value.

Substitution of Eq. (2) into the explicit expression for $\tilde{f}_{1}$ [see Eq. (2.52) in [1]] gives the following expression for the evolution of the normalized density modulation amplitude,

$$
\hat{f}_{1}(\hat{z})=\frac{\hat{F}^{\prime}(\hat{P}) \tilde{E}(\hat{z})}{\lambda_{1}+i(\hat{P}+\hat{C})}+\hat{f}_{1 \mid \hat{z}=0} \exp [-i \hat{z}(\hat{P}+\hat{C})],
$$

where $\lambda_{1}$ is the high-gain solution of the eigenvalue equation:

$$
\lambda_{j}-\hat{\mathcal{D}}=0 .
$$

Once an initial modulation $\hat{f}_{1 \mid \hat{z}=0}$ and an initial field amplitude $E_{0}$ are given, Eqs. (2) and (5) describe the evolution of the system.

\section{B. Optical klystron enhancement factor}

We now consider the effect of a dispersive element. We begin by discussing the impact of a detuned undulator, and we indicate with the phase $\Delta \psi$ the product of the frequency $\omega$ with the delay accumulated by an electron with respect to the wave:

$$
\Delta \psi=-\omega\left(\frac{1}{\left\langle v_{z}(\mathcal{E})\right\rangle}-\frac{1}{c}\right) \Delta z \simeq-\frac{\omega \Delta z}{2\left\langle\gamma_{z}(\mathcal{E})\right\rangle^{2} c},
$$

where $\Delta z$ is the longitudinal extension of the detuned section, $\left\langle v_{z}(\mathcal{E})\right\rangle$ is the average electron velocity for an electron with energy $\mathcal{E}$ and $\left\langle\gamma_{z}\right\rangle$ the corresponding longitudinal Lorentz factor. This definition fixes a convention such that $\Delta \psi>0$ indicates a delay. Expanding around the nominal energy $\mathcal{E}_{0}$ and remembering that $v_{z} \simeq c$, we obtain

$$
\Delta \psi \simeq-\frac{\omega \Delta z}{2\left\langle\gamma_{z}\left(\mathcal{E}_{0}\right)\right\rangle^{2} c}+C \Delta z+\frac{\omega P}{c \mathcal{E}_{0}} \frac{\Delta z}{\left\langle\gamma_{z}\left(\mathcal{E}_{0}\right)\right\rangle^{2}} .
$$

The longitudinal dispersion, identified by the $R_{56}$ element of the transfer matrix is just $R_{56}=\Delta z /\left\langle\gamma_{z}\left(\mathcal{E}_{0}\right)\right\rangle^{2}$. According to the previous convention, a positive $R_{56}$ means that particles with lower energies acquire a larger delay compared to those with higher energies.

We now consider the system in Fig. 1. We indicate with $\hat{z}_{1}$ the position at the exit of U1, so that Eqs. (2) and (5) with $\hat{z}=\hat{z}_{1}$ describe the system at that location. If we indicate with $R_{56}$ the dispersion due to the chicane and the phase 
shifter, instead of that due to the detuned undulator introduced above, our equations hold anyway. Following the notation in [2] here we introduce the parameter $D \equiv R_{56} \rho \omega / c$. Moreover, we define $\phi \equiv D /(2 \rho)$. In this way

$$
\Delta \psi \simeq+D(\hat{C}+\hat{P})-\phi .
$$

This means that, after the passage through chicane and phase shifter, the longitudinal phase space distribution of the electron bunch, $\hat{f}(\hat{P}, \psi)$ transforms to $\hat{f}(\hat{P}, \psi-\Delta \psi)$. We remind that $\hat{f}$ is related to $\hat{f}_{1}$, defined above, by the relation $\hat{f}(\hat{P}, \psi)=\hat{f}_{0}(\hat{P})+\hat{f}_{1}(\hat{P}) \exp (i \psi)+\hat{f}_{1}^{*}(\hat{P}) \exp (-i \psi)$. This means ${ }^{2}$ that $\hat{f}_{1}(\hat{P})$ transforms to $\hat{f}_{1}(\hat{P}) \exp (-i \Delta \psi)$, where $\Delta \psi$ is specified by Eq. (9). Therefore, if we indicate with $\hat{z}=\hat{z}_{1}^{+}$the position just after the dispersive elements, we find

$\tilde{E}\left(\hat{z}_{1}^{+}\right)=\left[\frac{E_{0}}{1-\hat{\mathcal{D}}_{\mid \lambda=\lambda_{1}}^{\prime}}+\frac{\hat{\mathcal{D}}_{f \mid \lambda=\lambda_{1}}}{1-\hat{\mathcal{D}}_{\mid \lambda=\lambda_{1}}^{\prime}}\right] \exp \left(\lambda_{1} \hat{z}_{1}\right)=\tilde{E}\left(\hat{z}_{1}\right)$,

because the dispersive elements have no effects on the field and

$$
\begin{aligned}
\hat{f}_{1}\left(\hat{z}_{1}^{+}\right)= & \frac{\hat{F}^{\prime}(\hat{P}) \tilde{E}(\hat{z})}{\lambda_{1}+i(\hat{P}+\hat{C})} \exp [i \phi-i D(\hat{C}+\hat{P})] \\
& +\hat{f}_{1 \mid \hat{z}=0} \exp \left[-i\left(D+\hat{z}_{1}\right)(\hat{P}+\hat{C})+i \phi\right] .
\end{aligned}
$$

Using the field and the bunching factor just after the dispersive elements, Eqs. (10) and (11), as initial conditions for the second undulator part, named U2 in Fig. 1, we can now find the field at the exit of $\mathrm{U} 2$ at position $\hat{z}=\hat{z}_{2}$ :

$\tilde{E}\left(\hat{z}_{2}\right)=\left[\frac{\tilde{E}\left(\hat{z}_{1}\right)}{1-\hat{\mathcal{D}}_{\mid \lambda=\lambda_{1}}^{\prime}}+\frac{\hat{\mathcal{D}}_{f \mid \lambda=\lambda_{1}}\left(\hat{z}_{1}^{+}\right)}{1-\hat{\mathcal{D}}_{\mid \lambda=\lambda_{1}}^{\prime}}\right] \exp \left[\lambda_{1}\left(\hat{z}_{2}-\hat{z}_{1}\right)\right]$.

The ratio $R$ of the field in the case of a given dispersion strength $D$ with the field at zero dispersion $D=0$ is known as optical klystron enhancement factor [2] and can be found by explicitly calculating Eq. (12) for the two cases of $D \neq 0$ and $D=0$, and taking the ratio of the two. It gives

\footnotetext{
${ }^{2}$ Note that we enforce the linear approximation, by which no harmonics except the first is generated. This implies that the relative energy modulation level $p_{m}$ at the dispersive element is such that $2 \pi R_{56} p_{m} / \lambda \ll 1$. However, we are not limiting the energy difference $P$ from reference. In fact, we will see that the phase $i D \hat{P}$, once integrated over the particle energy distribution leads to the parameter $D \hat{\Lambda}_{T}=2 \pi R_{56} \sigma_{\delta} / \lambda$, which varies freely to values larger than unity as we scan the chicane strength. This is a consequence of the dependence of $\Delta \psi$ on $\hat{P}$. Because of it, the chicane cannot be considered as a simple delay, as is the case, instead, of the small phase-shifter phase change, $\phi$.
}

$$
R=\frac{1+\int_{-\infty}^{\infty} \mathrm{d} \hat{P}^{\frac{\hat{F}^{\prime}(\hat{P}) \exp [-i(\hat{P}+\hat{C}) D+i \phi]}{\left[\lambda_{1}+i(\hat{P}+\hat{C})\right]^{2}}}}{1+\int_{-\infty}^{\infty} \mathrm{d} \hat{P} \frac{\hat{F}^{\prime}(\hat{P})}{\left[\lambda_{1}+i(\hat{P}+\hat{C})\right]^{2}}}
$$

This expression is basically ${ }^{3}$ equivalent to that in Eq. (2) of [2], which was derived for the first time in Eq. (7) of [5].

Finally, we note that $\hat{F}(\hat{P})$ must be vanishing for $\hat{P} \rightarrow \pm \infty$. Then, integration by parts yields the following expression equivalent to Eq. (13):

$$
R=\frac{1-\int_{-\infty}^{\infty} \mathrm{d} \hat{P} \frac{\hat{F}(\hat{P}) \exp [-i(\hat{P}+\hat{C}) D+i \phi]}{\left[\lambda_{1}+i(\hat{P}+\hat{C})\right]^{2}}\left\{\frac{-2 i}{\left[\lambda_{1}+i(\hat{P}+\hat{C})\right]-i D}\right\}}{1+2 i \int_{-\infty}^{\infty} \mathrm{d} \hat{P} \frac{\hat{F}(\hat{P})}{\left[\lambda_{1}+i(\hat{P}+\hat{C})\right]^{3}}}
$$

\section{Optical klystron power gain factor}

The klystron power gain factor is defined in [2] by integrating the square modulus of $\mathrm{R}$ over the FEL spectrum $S(\hat{C})$ :

$$
G=\int_{-\infty}^{\infty} \mathrm{d} \hat{C}|R(\hat{C})|^{2} S(\hat{C})
$$

In the following we will assume an average selfamplified spontaneous emission (SASE) spectrum

$$
S(\hat{C})=\frac{1}{\sqrt{2 \pi} \sigma_{\hat{C}}} \exp \left[-\frac{\left(\hat{C}-\hat{C}_{m}\right)^{2}}{2 \sigma_{\hat{C}}^{2}}\right],
$$

where $\hat{C}_{m}$ indicated the optimal detuning from resonance. Moreover, from now on we will assume

$$
\hat{F}(\hat{P})=\frac{1}{\sqrt{2 \pi} \hat{\Lambda}_{T}} \exp \left(-\frac{\hat{P}^{2}}{2 \hat{\Lambda}_{T}^{2}}\right) .
$$

We note that with these definitions, the physical meaning of $\hat{\Lambda}_{T}$ is that of the usual normalized energy spread parameter: $\hat{\Lambda}_{T}=\sigma_{\delta} / \rho$, with $\sigma_{\delta}=\sqrt{\left\langle(\delta \mathcal{E})^{2}\right\rangle} / \mathcal{E}_{0}$.

\section{Limit for $\hat{\Lambda}_{T} \gg 1$}

When $\hat{\Lambda}_{T} \gg 1, \hat{F}(\hat{P})$ is about constant, and therefore $\hat{F}^{\prime}(\hat{P}) \simeq 0$. From Eq. (13), one readily has $R \simeq 1$. This limit has no practical importance for FELs.

\footnotetext{
${ }^{3}$ In order to see this, the reader should substitute notations: $\hat{F} \rightarrow V, \hat{P} \rightarrow \xi, \hat{C} \rightarrow(1-\nu) /(2 \rho), \lambda_{1}+i \hat{C} \rightarrow-i \mu$. By doing so, one only finds a difference in the phase factor, where one of the phase terms in the numerator reads $i \rho R_{56} k_{r} \xi$ in our expression, instead of $i \rho R_{56} k_{r} \xi \nu$ in [2]. The difference is unimportant as $\nu=$ $\omega / \omega_{r} \simeq 1$ with $\omega_{r}=c k_{r}$ is the resonant frequency.
} 


\section{E. Limit for $\hat{\Lambda}_{T} \ll 1$}

The limit for $\hat{\Lambda}_{T} \ll 1$ is of practical importance for a number of FELs. Since energy spread effects become important when $D$ is comparable with $\hat{\Lambda}_{T}^{-1}$, in this limiting case the expression for the power gain factor $G$ includes simultaneously a small, $\hat{\Lambda}_{T}$, and a large, $D$, parameter. As a result, care must be taken while controlling the accuracy of the calculations.

Let us consider Eq. (14). Once the integration in $\hat{P}$ is performed, one obtains terms of $R$ where different orders in $D$ and $\hat{\Lambda}_{T}$ mix up. In order to control the accuracy of $R$ one needs to count $D$ of order $\hat{\Lambda}_{T}^{-1}$, and expand $R$ accordingly. When discussing about a certain order in $\hat{\Lambda}_{T}$ accounting for $D$ as of order $\hat{\Lambda}_{T}^{-1}$, we will talk about the effective order in $\hat{\Lambda}_{T}$. To be explicit, a term proportional to $D^{m} \hat{\Lambda}_{T}^{n}$ is of effective order $n-m$ in $\hat{\Lambda}_{T}$.

In order to calculate $G$, one then needs to take the square modulus of $R$. If a defined accuracy to a certain fixed order in $\hat{\Lambda}_{T}$ is wanted, special care must be taken to include all relevant terms. For example, in the expression for $R$, any linear term in $D$ [like the term $-i D$ in curled brackets in Eq. (14)] and any third order term in $\hat{\Lambda}_{T}$ will yield, in the expression for $G$, to a term of the second order in $\hat{\Lambda}_{T}$. As a result, accounting for $D$ as of order $\hat{\Lambda}_{T}^{-1}$, an expansion of $R$ up to the second effective order in $\hat{\Lambda}_{T}$ is not sufficient to obtain a consistent expansion of $G$ up to the second effective order in $\hat{\Lambda}_{T}$.

When expanding $R$ and $G$, one should consider that the eigenvalue $\lambda_{1}$ depends both on $\hat{\Lambda}_{T}$ and on the detuning parameter $\hat{C}$, i.e., $\lambda_{1}=\lambda_{1}\left(\hat{C}, \hat{\Lambda}_{T}\right)$, and must be found explicitly by solving the eigenvalue equation. The detuning parameter can be further expanded, independently, to a fixed order around the value corresponding to the optimum amplification $\hat{C}_{m}$, which in its turn depends on $\hat{\Lambda}_{T}$, i.e., $\hat{C}_{m}=\hat{C}_{m}\left(\hat{\Lambda}_{T}\right)$. Note that for ordering purposes, we can consider $\lambda_{1}$ of order unity.

\section{Expansion of $\boldsymbol{R}$ in $\hat{\boldsymbol{P}}$ around zero}

Having made these preliminary considerations, we consider the integration in $\hat{P}$ in Eq. (14). The distribution $\hat{F}(\hat{P})$ becomes very narrow (order $\hat{\Lambda}_{T} \ll 1$ ) with respect to the integrand. In other words, we are integrating over values of $\hat{P}$ near to zero. This allows an expansion of all the terms in $\hat{P}$ under the integral with exception of $F(\hat{P}) \exp (-i \hat{P} D)$ in the numerator and $F(\hat{P})$ in the denominator.

Consider first the denominator. Due to the symmetry of the distribution in Eq. (17) odd orders in $\hat{P}$ yield zero contribution. A second (or third) order expansion in $\hat{P}$ gives

$$
\text { Denominator of } \mathrm{R}=1+\frac{2 i\left[\left(i \hat{C}+\lambda_{1}\right)^{2}-6 \hat{\Lambda}_{T}^{2}\right]}{\left(i \hat{C}+\lambda_{1}\right)^{5}} \text {. }
$$

Therefore, the denominator includes terms of order unity and smaller, and $D$ does not enter in it. The difference with respect to a first order expansion in $\hat{P}$ is the term in $\hat{\Lambda}_{T}^{2}$. Expanding to the fourth and higher orders in $\hat{P}$ would lead to corrections of the fourth order and higher in $\hat{\Lambda}_{T}$.

Consider now the numerator. Direct calculations show that the second order expansion in $\hat{P}$ differs from the first order expansion in $\hat{P}$ of terms of effective order $\hat{\Lambda}_{T}$ and smaller. The third order expansion differs from the second order of terms of effective order $\hat{\Lambda}_{T}^{2}$ and smaller. The fourth order expansion differs from the third order of terms of effective order $\hat{\Lambda}_{T}^{3}$ and smaller, and so on. This is due to the mathematical structure of the integral. In fact, terms of order $n$ in $\hat{P}$ give, after integration, dominant terms of effective order $\hat{\lambda}_{T}^{n-1}$. This means that the $n$th order expansion in $\hat{P}$ includes all terms of effective order $\hat{\Lambda}_{T}^{n-1}$.

Once an effective order in $\hat{\Lambda}_{T}$ is fixed, as a result of the expansion in $\hat{P}$, integration of the numerator in Eq. (14) will involve integrals of the form $\int_{-\infty}^{\infty} \mathrm{d} \hat{P} \hat{P}^{k} \hat{F}(\hat{P}) \exp (-i \hat{P} D)$, with $k$ a positive integer, which will result in the following form of Eq. (14):

$$
R=f_{1}\left(\hat{\Lambda}_{T}, \hat{C}\right)+f_{2}\left(\hat{\Lambda}_{T}, \hat{C}\right) \exp \left(-\frac{D^{2} \hat{\Lambda}_{T}^{2}}{2}\right)
$$

so that

$$
\begin{aligned}
G \equiv & G_{1}+G_{2}+G_{3} \\
= & \int_{-\infty}^{\infty} \mathrm{d} \hat{C}\left|f_{1}\left(\hat{\Lambda}_{T}, \hat{C}\right)\right|^{2} S(\hat{C}) \\
& +\int_{-\infty}^{\infty} \mathrm{d} \hat{C}\left|f_{2}\left(\hat{\Lambda}_{T}, \hat{C}\right)\right|^{2} S(\hat{C}) \exp \left(-D^{2} \hat{\Lambda}_{T}^{2}\right) \\
& +\int_{-\infty}^{\infty} \mathrm{d} \hat{C}\left[f_{1}\left(\hat{\Lambda}_{T}, \hat{C}\right) \bar{f}_{2}\left(\hat{\Lambda}_{T}, \hat{C}\right)\right. \\
& \left.+\bar{f}_{1}\left(\hat{\Lambda}_{T}, \hat{C}\right) f_{2}\left(\hat{\Lambda}_{T}, \hat{C}\right)\right] S(\hat{C}) \exp \left(-\frac{D^{2} \hat{\Lambda}_{T}^{2}}{2}\right) .
\end{aligned}
$$

This derivation highlights the meaning of the different dependencies on $D$ of the various terms $G_{1}, G_{2}$ and $G_{3}$ as a consequence of the square modulus operation. In particular, one sees a first term, undamped, a second term damped according to $\exp \left(-D^{2} \hat{\Lambda}_{T}^{2}\right)$ and a third interference term arising damped according to $\exp \left(-D^{2} \hat{\Lambda}_{T}^{2} / 2\right)$. This last term includes oscillations due to the phase in $f_{2}$.

Since $R$ is expanded to a given order in $\hat{C}$ around the optimum amplification value $\hat{C}_{m}\left(\hat{\Lambda}_{T}\right)$, the accuracy of the final result depends on the actual spectral width and on the order of the expansion in $\hat{C}$. In the limit for $\sigma_{\hat{C}} \rightarrow 0$, for example, a zeroth order expansion in $\hat{C}$ around $\hat{C}_{m}\left(\hat{\Lambda}_{T}\right)$ 
will be sufficient. However, in practical cases, $\sigma_{\hat{C}}$ is of order unity ${ }^{4}$ and keeping higher orders is necessary. Note that while $\hat{C}_{m}$ is (see the next paragraph) of order $\hat{\Lambda}_{T}^{2}$, when performing the various expansions we should consider $\hat{C}$ of order unity in order to be able to treat cases when $\sigma_{\hat{C}}$ is of order unity.

\section{Eigenvalue equation}

Before we proceed further, we need to find an expression for $\lambda_{1}\left(\hat{C}, \hat{\Lambda}_{T}\right)$, i.e., we need to solve the FEL eigenvalue equation. Here we introduce corrections to the cold-beam case, to the second order in $\hat{\Lambda}_{T}$. We follow [1] and report the following eigenvalue equation in the case of Gaussian energy spread, Eq. (17), which is valid in the high-gain limit, for $\operatorname{Re}\left(\lambda_{1}\right)>0$ :

$$
\lambda_{1}=i \int_{0}^{\infty} \mathrm{d} \xi \xi \exp \left[-\frac{\hat{\Lambda}_{T}^{2} \xi^{2}}{2}-\left(\lambda_{1}+i \hat{C}\right) \xi\right] .
$$

When $\hat{\Lambda}_{T}=0$, Eq. (21) becomes the usual cubic equation for the cold case

$$
\lambda=i(\lambda+i \hat{C})^{-2}
$$

where the high-gain solution can be written explicitly as

$$
\begin{aligned}
\lambda_{0}= & \frac{1}{3}\left[-2 i \hat{C}-\frac{2^{1 / 3} \hat{C}^{2}}{\left(27 i-2 i \hat{C}^{3}+3 \sqrt{3} \sqrt{-27+4 \hat{C}^{3}}\right)^{1 / 3}}\right. \\
& \left.+\frac{\left(27 i-2 i \hat{C}^{3}+3 \sqrt{3} \sqrt{-27+4 \hat{C}^{3}}\right)^{1 / 3}}{2^{1 / 3}}\right] .
\end{aligned}
$$

Since we deal with $\hat{\Lambda}_{T} \ll 1$, we can find a correction $\delta \lambda$ to $\lambda_{0}$ up to the second order in $\hat{\Lambda}_{T}$ by approximating Eq. (21) as

$$
\lambda_{0}+\delta \lambda=i \int_{0}^{\infty} \mathrm{d} \xi \xi\left(1-\frac{\hat{\Lambda}_{T}^{2} \xi^{2}}{2}\right) \exp \left[-\left(\lambda_{0}+i \hat{C}\right) \xi\right](1-\xi \delta \lambda),
$$

where we assumed $\exp (-\xi \delta \lambda) \simeq(1-\xi \delta \lambda)$. Equation (24) can be cast in the form of an equation for $\delta \lambda$, with solution

$$
\delta \lambda=-\frac{3 i \hat{\Lambda}_{T}^{2}}{\left(i \hat{C}+\lambda_{0}\right)^{4}\left[1+\frac{2 i}{\left(i \hat{C}+\lambda_{0}\right)^{3}}\right]} .
$$

This solution is consistent with our choice of accuracy to the second order in $\hat{\Lambda}_{T}$. The eigenvalue is thus

$$
\lambda_{1} \simeq \lambda_{0}+\frac{3 i \hat{\Lambda}_{T}^{2} \lambda_{0}^{2}}{\left[1+\frac{2 i}{\left(i \hat{C}+\lambda_{0}\right)^{3}}\right]} .
$$

Equation (26) can now be substituted into Eqs. (7) and (8) [or in principle directly into Eq. (30)]. Before doing so, we note that we have now separate control on the accuracy that we want to keep for the detuning parameter $\hat{C}$. For example, if we keep up to the second order in $\hat{C}$, the expression for Eq. (26) becomes

$$
\begin{aligned}
\lambda_{1} \simeq & \left(\frac{\sqrt{3}}{2}-\frac{\hat{C}^{2}}{6 \sqrt{3}}\right)+i\left(\frac{1}{2}-\frac{2}{3} \hat{C}+\frac{\hat{C}^{2}}{18}\right) \\
& +\left[\left(-\frac{\sqrt{3}}{2}+\frac{\hat{C}}{\sqrt{3}}\right)+i\left(\frac{1}{2}+\frac{\hat{C}}{3}\right)\right] \hat{\Lambda}_{T}^{2} .
\end{aligned}
$$

The next order correction is of order $\hat{\Lambda}_{T}^{4}$. For our purposes we will not need to calculate it explicitly, because terms related with this correction will drop out of actual calculations, but we will still indicate it as $\beta_{1} \hat{\Lambda}_{T}^{4}$, with $\beta_{1}$ a complex number.

Since we deal with the high-gain limit, only values of $\hat{C}$ near to the optimum value should be accounted for. It has been shown (see [1], paragraph 2.1.3) that the optimum detuning in the limit of a small energy spread is given by $\hat{C}_{m} \simeq 3 \hat{\Lambda}_{T}^{2}$. We can indicate the next order correction to the optimum detuning value with $\beta_{2} \hat{\Lambda}_{T}^{4}$, with $\beta_{2}$ a real number. Terms related with this correction also drop out of explicit calculations.

\section{Expansion of $\boldsymbol{R}$ in $\hat{\boldsymbol{P}}$ around zero}

We can now begin to consider a second order expansion of the integrands Eq. (14) in $\hat{P}$, which means we are accounting for all terms of effective order up to $\hat{\Lambda}_{T}$. The full expression for the numerator of $R$ is

$$
\begin{aligned}
\text { Numerator of } \mathrm{R}= & 1-\exp \left(-\frac{D^{2} \hat{\Lambda}_{T}^{2}}{2}-i \hat{C} D+i \phi\right)\left[\frac{-2 i+\hat{C} D-i D \lambda_{1}}{\left(i \hat{C}+\lambda_{1}\right)^{3}}\right. \\
& \left.+\frac{i \hat{\Lambda}_{T}^{2}\left[12-i D\left(i \hat{C}+\lambda_{1}\right)\left(9 i-2 \hat{C} D+2 i D \lambda_{1}\right)\right]+3 i D^{2}\left(-4-i \hat{C} D-\lambda_{1} D\right) \hat{\Lambda}_{T}^{4}}{\left(i \hat{C}+\lambda_{1}\right)^{5}}\right] .
\end{aligned}
$$

${ }^{4}$ The reader can verify that $\sigma_{\hat{C}}=1 / 2$ corresponds to a spectral width of $\rho$, which is of order of the FEL bandwidth. 
Since the second order expansion includes all terms of effective order up to $\hat{\Lambda}_{T}$, we can neglect terms that contain only higher effective orders. With already in mind the expressions for $\lambda_{1}$ and for the optimum value of $\hat{C}$ we can neglect a few terms and write

$$
\begin{aligned}
\text { Numerator of } \mathrm{R}= & 1-\exp \left(-\frac{D^{2} \hat{\Lambda}_{T}^{2}}{2}-i \hat{C} D+i \phi\right)\left[\frac{-2 i+\hat{C} D-i D \lambda_{1}}{\left(i \hat{C}+\lambda_{1}\right)^{3}}\right. \\
& \left.+\frac{i \hat{\Lambda}_{T}^{2}\left[-i D\left(i \hat{C}+\lambda_{1}\right)\left(9 i-2 \hat{C} D+2 i D \lambda_{1}\right)\right]+3 i D^{2}\left(-i \hat{C} D-\lambda_{1} D\right) \hat{\Lambda}_{T}^{4}}{\left(i \hat{C}+\lambda_{1}\right)^{5}}\right] .
\end{aligned}
$$

Using Eqs. (18) and (29) we see that Eq. (14) gives

$$
\begin{aligned}
R= & \left\{1+\frac{2 i\left[\left(i \hat{C}+\lambda_{1}\right)^{2}-6 \hat{\Lambda}_{T}^{2}\right]}{\left(i \hat{C}+\lambda_{1}\right)^{5}}\right\}^{-1}\left\{1-\exp \left(-\frac{D^{2} \hat{\Lambda}_{T}^{2}}{2}-i \hat{C} D+i \phi\right)\left[\frac{-2 i+\hat{C} D-i D \lambda_{1}}{\left(i \hat{C}+\lambda_{1}\right)^{3}}\right.\right. \\
& \left.\left.+\frac{i \hat{\Lambda}_{T}^{2}\left[-i D\left(i \hat{C}+\lambda_{1}\right)\left(9 i-2 \hat{C} D+2 i D \lambda_{1}\right)\right]+3 i D^{2}\left(-i \hat{C} D-\lambda_{1} D\right) \hat{\Lambda}_{T}^{4}}{\left(i \hat{C}+\lambda_{1}\right)^{5}}\right]\right\} .
\end{aligned}
$$

We now define the two auxiliary functions

$$
f_{1}(\hat{C})=\left\{1+\frac{2 i\left[\left(i \hat{C}+\lambda_{1}\right)^{2}-6 \hat{\Lambda}_{T}^{2}\right]}{\left(i \hat{C}+\lambda_{1}\right)^{5}}\right\}^{-1}
$$

and

$f_{2}(\hat{C})=-f_{1}(\hat{C}) \exp (-i \hat{C} D+i \phi)\left[\frac{-2 i+\hat{C} D-i D \lambda_{1}}{\left(i \hat{C}+\lambda_{1}\right)^{3}}+\frac{i \hat{\Lambda}_{T}^{2}\left[-i D\left(i \hat{C}+\lambda_{1}\right)\left(9 i-2 \hat{C} D+2 i D \lambda_{1}\right)\right]+3 i D^{2}\left(-i \hat{C} D-\lambda_{1} D\right) \hat{\Lambda}_{T}^{4}}{\left(i \hat{C}+\lambda_{1}\right)^{5}}\right]$

In order to calculate $f_{1}$ and $f_{2}$ explicitly we use Eq. (27). Since we deal with the high-gain limit we can effectively expand around $\hat{C}=\hat{C}_{m} \simeq 3 \hat{\Lambda}_{T}^{2}$, except in the phase factor of $f_{2}$. The order of this expansion is a fully independent parameter. Finally, we expand in $\hat{\Lambda}_{T}$, keeping only up to the first effective order.

Expanding to the zeroth order in $\hat{C}$ we obtain

$$
f_{1}=\frac{1}{3}
$$

and

$$
\begin{aligned}
f_{2}= & \frac{1}{6} \exp (-i \hat{C} D+i \phi) \\
& \times\left[(i+\sqrt{3}) D+4\left(1-D^{2} \hat{\Lambda}_{T}^{2}\right)\right. \\
& \left.+(\sqrt{3}-i)\left(-5 D \hat{\Lambda}_{T}+3 D^{3} \hat{\Lambda}_{T}^{3}\right) \hat{\Lambda}_{T}\right] .
\end{aligned}
$$

We can use these expressions for $f_{1}$ and $f_{2}$ to calculate $G_{1}$, $G_{2}$ and $G_{3}$. Note that the zeroth order expansion in $\hat{C}$ around $\hat{C}_{m}$ is equivalent to the substitution $\hat{C}=\hat{C}_{m} \simeq 3 \hat{\Lambda}_{T}^{2}$, except for the phase factor of $f_{2}$, which we did not expand because it is highly oscillatory. Finally, we must remember from the previous discussion that $f_{1}$ and $f_{2}$ include all terms up to the first order, but that they also include terms of order $\hat{\Lambda}_{T}^{-1}$. When calculating the square modulus, the terms of order $\hat{\Lambda}_{T}^{-1}$ mix with the others: if we had kept terms up to $\hat{\Lambda}_{T}^{2}$, the mixed terms would give terms of order $\hat{\Lambda}_{T}$. Therefore, if we want a well-defined expansion order in $\hat{\Lambda}_{T}$, we must only keep terms to the zero order in $\hat{\Lambda}_{T}$, which gives

$$
\begin{gathered}
G_{1}=\frac{1}{9} \\
G_{2}=\frac{1}{9} \exp \left(-D^{2} \hat{\Lambda}_{T}^{2}\right)\left[4+2 \sqrt{3} D+D^{2}-13 D^{2} \hat{\Lambda}_{T}^{2}\right. \\
\left.-2 \sqrt{3} D^{3} \hat{\Lambda}_{T}^{2}+7 D^{4} \hat{\Lambda}_{T}^{4}\right]
\end{gathered}
$$

$$
\begin{aligned}
G_{3}= & \frac{1}{9} \exp \left[-\frac{D^{2}\left(\hat{\Lambda}_{T}^{2}+\sigma_{\hat{C}}^{2}\right)}{2}\right] \\
& \times\left[\left(4+\sqrt{3} D-4 D^{2} \hat{\Lambda}_{T}^{2}\right) \cos (\phi)-D \sin (\phi)\right],
\end{aligned}
$$


where we neglect the term $-3 D \hat{\Lambda}_{T}^{2}$ in the argument of the sinusoidal functions in agreement with our prescription of keeping, effectively, zeroth-order terms in $\hat{\Lambda}_{T}$. The dependence on $\sigma_{\hat{C}}$ follows from the only dependence on $\hat{C}$ explicitly left in $f_{2}$, which is in the phase.

An expression for $G$ to the zeroth effective order in $\hat{\Lambda}_{T}$ but to the second order in $\hat{C}$, and an expression for $G$ to the second effective order in $\hat{\Lambda}_{T}$ and the zeroth order in $\hat{C}$ are explicitly given in the Appendix of this paper. This last expression is found upon an expansion of $R$ to the third effective order in $\hat{\Lambda}_{T}$.

\section{NUMERICAL CALCULATIONS AND RESULTS}

In the previous section we considered a derivation of $R$ and $G$ in the limit for $\hat{\Lambda}_{T} \ll 1$. However, in both cases we relied on $\hat{\Lambda}_{T} \ll 1$, while in cases of practical interest we deal with small values of $\hat{\Lambda}_{T}$, but not necessarily with the limiting case for a vanishing small $\hat{\Lambda}_{T} \ll 1$. While, in principe, one can expand the expression for $G$ to higher orders in $\hat{\Lambda}_{T}$, in practice the analytical results become more and more complicated. As a result, numerical calculations are simpler to perform, in order to improve accuracy of the results.

Numerical calculations can be performed using directly Eqs. (13) and (15). First, the eigenvalue equation is solved numerically for a Gaussian energy spread profile, in the limit for a high-gain amplifier. The equation to be solved, see [1], is

$$
\lambda_{1}=i \int_{0}^{\infty} \mathrm{d} \xi \xi \exp \left[-\frac{\hat{\Lambda}_{T}^{2} \xi^{2}}{2}-\left(\lambda_{1}+i \hat{C}\right) \xi\right] .
$$

While solving Eq. (38), special care must be taken with the initial value of the root-finding routine. We start with the maximum value of $\hat{C}$, giving the cold solution as an initial condition, and we feed the newly found root as an initial value for the next step. Solutions for the eigenvalue equations are rendered graphically in Fig. 2, where they are compared with the cold-beam case. Note that Eq. (38) gives acceptable values only for $\hat{C}$ corresponding to $\operatorname{Re}\left(\lambda_{1}\right)>0$.

Once $\lambda_{1}(\hat{C})$ is tabulated for a certain value of $\hat{\Lambda}_{T}$, we tabulate $R$ as a function of $\hat{C}$ by evaluating numerically the numerator and the denominator of Eq. (13), using as integration limits $\pm 5 \hat{\Lambda}_{T}$. Finally, we use $R(\hat{C})$, integrating Eq. (15) for limiting values of $\hat{C}$ depending on $\sigma_{\hat{C}}$. In particular we used $\hat{C}$ between \pm 2.5 for $\sigma_{\hat{C}}=0.1$, and $\hat{C}$ between \pm 4 for $\sigma_{\hat{C}}=0.2, \sigma_{\hat{C}}=0.5$. This is more than enough to include the high-gain case for moderate levels of $\hat{\Lambda}_{T}$, which is what we are interested in for the case of a working FEL. Finally, it should be reminded that, when one calculates $G$, special care is needed when integrating numerically in $\hat{C}$. In fact, our solutions for $\lambda_{1}$ [Eq. (21)] is correct only for $\operatorname{Re}\left(\lambda_{1}\right)>0$. Therefore, integrating for $\hat{C}$ between \pm 4 is only justified under the assumption of the high-gain limit that bounds the effective values of the detuning. While performing the numerical calculations, one should be careful with the point around $\hat{C}_{0}=3 / 2^{2 / 3} \simeq 1.89$. At that position for a cold beam (and around that position for nonzero energy spread), the real part of $\lambda_{1}$ becomes zero, and the denominator in Eq. (13) also vanishes, leading to numerical difficulties. For relatively small values of $\sigma_{\hat{C}}$ this is not a problem because the contribution to $\mathrm{G}$ around $\hat{C}_{0}$ is damped exponentially.

Once $G_{1}, G_{2}$ and $G_{3}$ are evaluated numerically, we can proceed to a comparison with the analytical expressions derived above, for the case $\hat{\Lambda}_{T} \ll 1$. In all these cases we identified, during our analysis, important differences with expressions in [2], which are traced back to the presence of a parameter, $D$, assuming large values up to order $\hat{\Lambda}_{T}^{-1}$. Following our notations, ${ }^{5}$ the expressions in [2] can be written as

$$
\begin{aligned}
G \simeq & \frac{1}{9}\left\{1+\left(4+D^{2}\right) \exp \left(-D^{2} \hat{\Lambda}_{T}^{2}\right)+2 \sqrt{3} D \exp \left(-\frac{D^{2} \hat{\Lambda}_{T}^{2}}{2}\right)\right. \\
& +[(4+\sqrt{3} D) \cos (\phi)-D \sin (\phi)] \\
& \left.\times \exp \left(\frac{-D^{2} \hat{\Lambda}_{T}^{2}}{2}\right) \exp \left(\frac{-D^{2} \sigma_{\hat{C}}^{2}}{2}\right)\right\} .
\end{aligned}
$$

Essentially, ${ }^{6}$ these expressions result from expansions that are carried out without accounting for the fact that $D$ can be large, resulting in the neglection of a few important terms. These terms are readily identified by comparison with our expressions, e.g., Eqs. (35)-(37).

\section{A. Comparison of $G$ using different expansions for different values of $\sigma_{\hat{C}}$}

In Figs. 3 and 4 we consider the two cases for $\sigma_{\hat{C}}=0.1$ and $\sigma_{\hat{C}}=0.5$ (this last value corresponding to a relative spectral width of order $\rho$ ). The different rows show different values of $\hat{\Lambda}_{T}$. The left column illustrates $G_{1}+G_{2}$, while the right column illustrates $G=G_{1}+G_{2}+G_{3}$. The blue lines refer to numerical integration, the black lines show the zeroth effective order in $\hat{\Lambda}_{T} \ll 1$, using a zeroth order expansion in $\hat{C}$ [Eqs. (35)-(37)]. The yellow dashed lines refer to the zeroth effective order in $\hat{\Lambda}_{T} \ll 1$, using a second order expansion in $\hat{C}$ [Eqs. (A3)-(A5)]. The red dashed lines illustrate expressions for the second effective order in

\footnotetext{
${ }^{5}$ In the original paper [2] $\sigma_{\xi}$ corresponds to $\hat{\Lambda}_{T}$, while $(1-$ $\nu) /(2 \rho)$ corresponds to our symbol $\hat{C}$, so that $\sigma_{\hat{C}}=\sigma_{\nu} /(2 \rho)$.

${ }^{6} \mathrm{~A}$ different question concerns the term $2 \sqrt{3} D \exp \left(-\frac{D^{2} \hat{\Lambda}_{T}^{2}}{2}\right)$, which should be part of $G_{2}$ and in our case actually reads $2 \sqrt{3} D \exp \left(-D^{2} \hat{\Lambda}_{T}^{2}\right)$, see Eq. (36), in agreement with the general shape of $G$ in Eq. (20).
} 

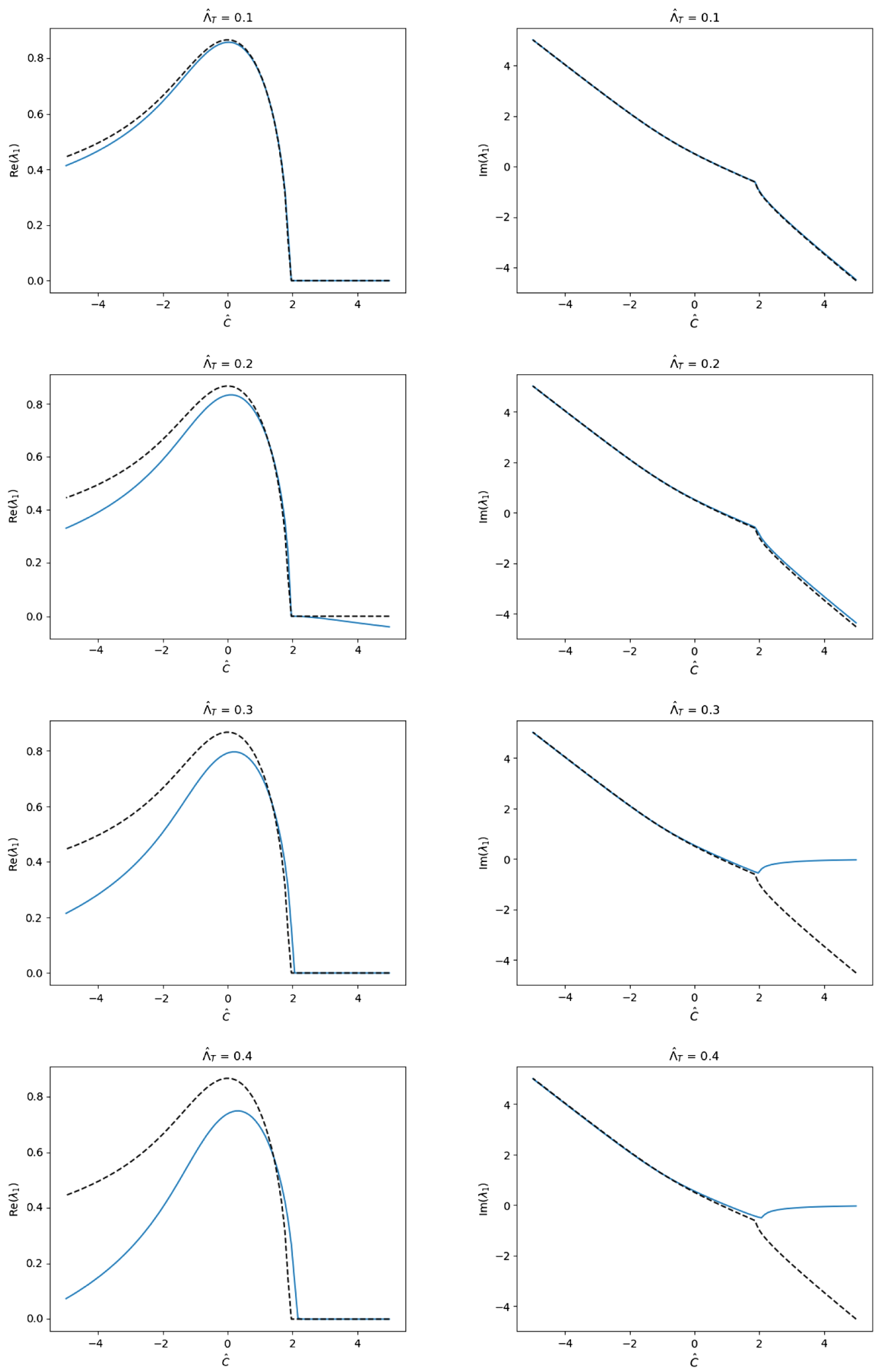

FIG. 2. Numerical solutions (blue lines) of the eigenvalue equation for different values of $\hat{\Lambda}_{T}$. The dashed line corresponds to the coldbeam case. Note that only the values for $\hat{C}$ corresponding to $\operatorname{Re}\left(\lambda_{1}\right)>0$ are mathematically acceptable. 

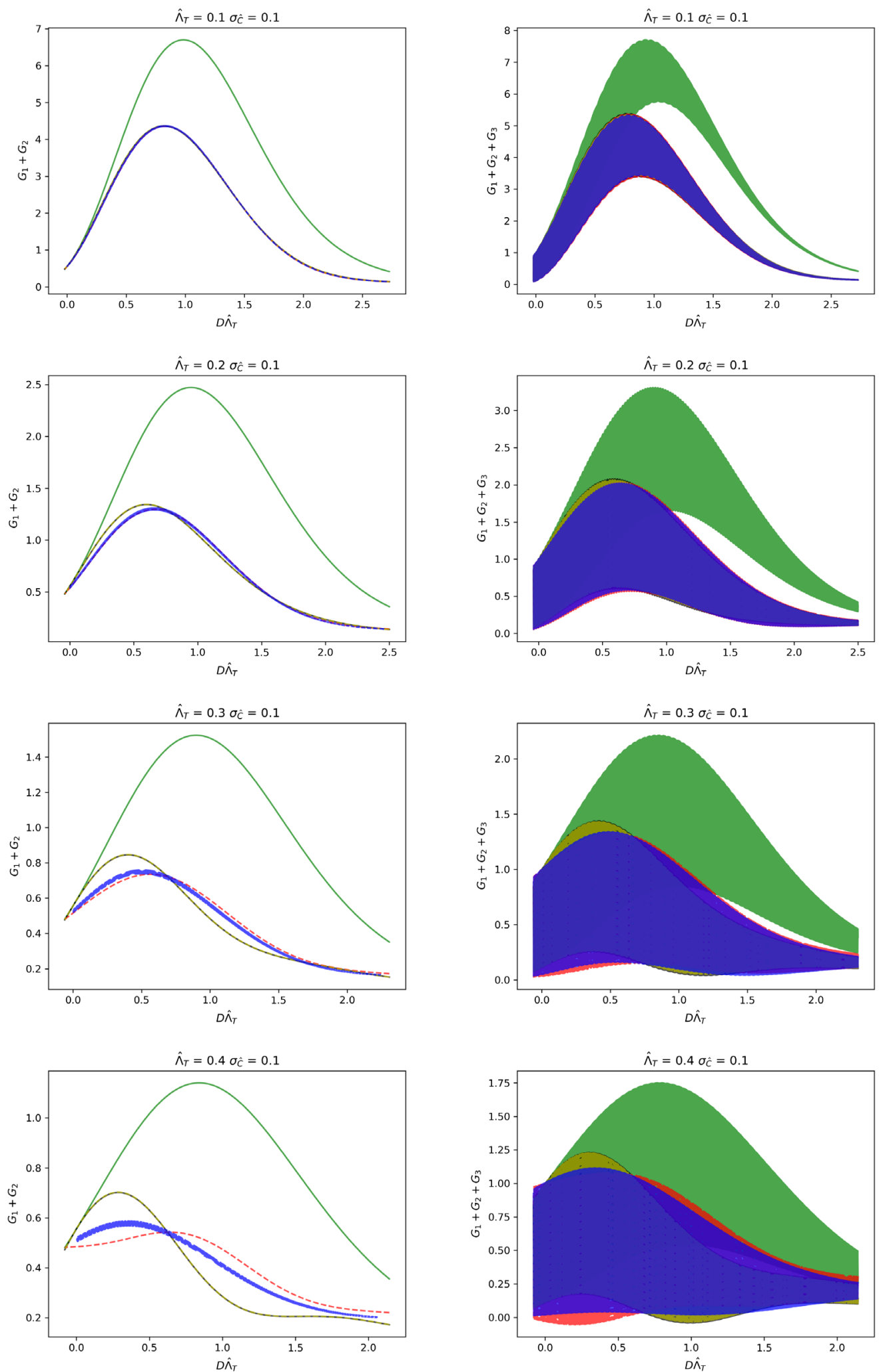

FIG. 3. $\sigma_{\hat{C}}=0.1$. Left column: comparison of $G_{1}+G_{2}$ at different values of $\hat{\Lambda}_{T}=0.1,0.2,0.3,0.4$ (rows one to four) using different methods discussed in this paper. Right column: comparison of $G_{1}+G_{2}+G_{3}$ at different values of $\hat{\Lambda}_{T}=0.1,0.2,0.3,0.4$ (rows one to four) using different methods discussed in this paper. These different methods are rendered in the following way. Blue lines: numerical integration; black lines: zeroth effective order in $\hat{\Lambda}_{T} \ll 1$, using a zeroth order expansion in $\hat{C}$ [Eqs. (35) and (36)]; yellow dashed lines: zeroth effective order in $\hat{\Lambda}_{T} \ll 1$, using a second order expansion in $\hat{C}$ [Eqs. (A3) and (A4)]; red dashed lines: second effective order in $\hat{\Lambda}_{T} \ll 1$, using a zeroth order expansion in $\hat{C}$ [Eqs. (A8) and (A9)]; green lines: results from Ref. [2]. 

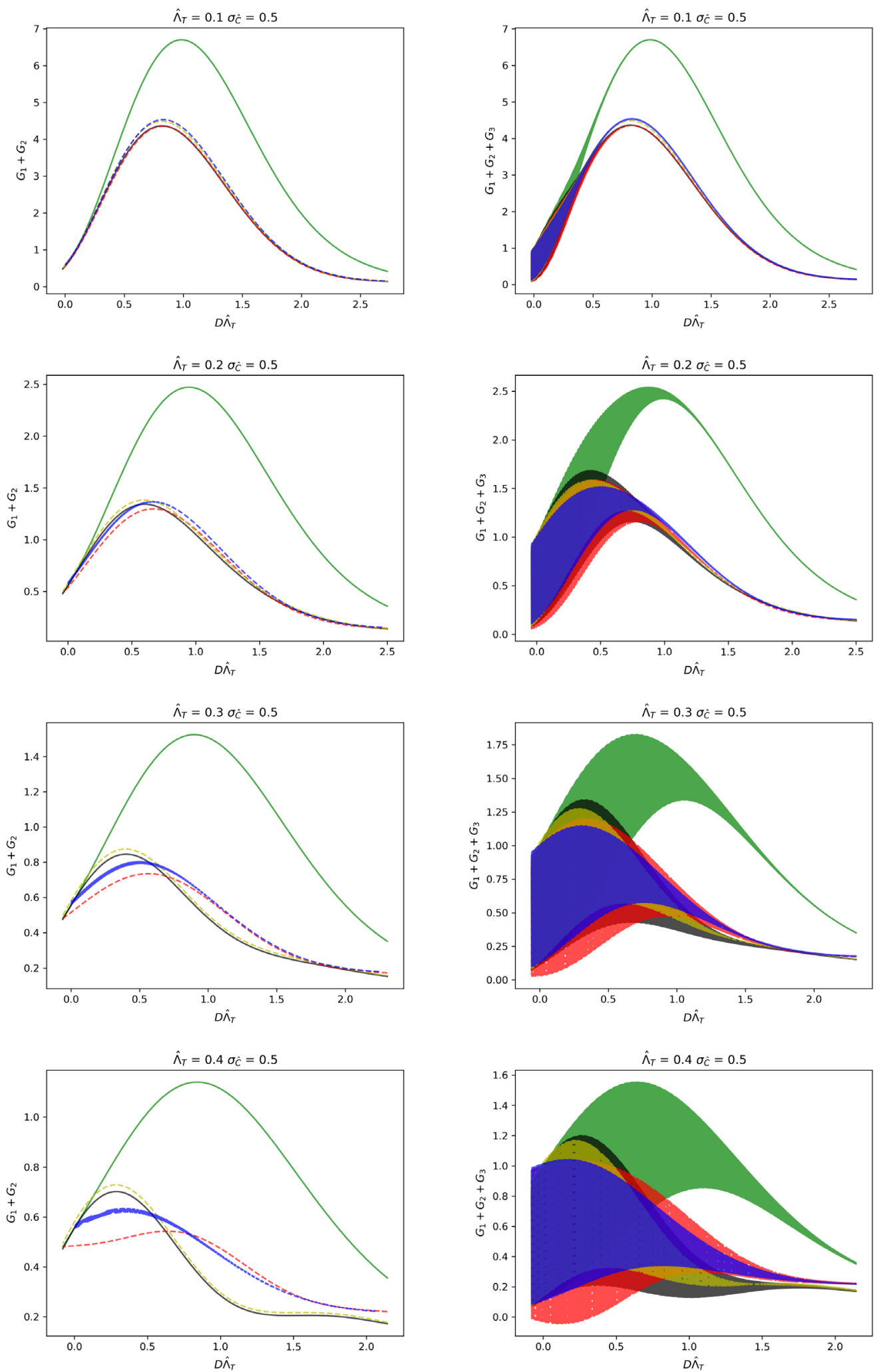

FIG. 4. $\sigma_{\hat{C}}=0.5$. Left column: comparison of $G_{1}+G_{2}$ at different values of $\hat{\Lambda}_{T}=0.1,0.2,0.3,0.4$ (rows one to four) using different methods discussed in this paper. Right column: comparison of $G_{1}+G_{2}+G_{3}$ at different values of $\hat{\Lambda}_{T}=0.1,0.2,0.3,0.4$ (rows one to four) using different methods discussed in this paper. These different methods are rendered in the following way. Blue lines: numerical integration; black lines: zeroth effective order in $\hat{\Lambda}_{T} \ll 1$, using a zeroth order expansion in $\hat{C}$ [Eqs. (35) and (36)]; yellow dashed lines: zeroth effective order in $\hat{\Lambda}_{T} \ll 1$, using a second order expansion in $\hat{C}$ [Eqs. (A3) and (A4)]; red dashed lines: second effective order in $\hat{\Lambda}_{T} \ll 1$, using a zeroth order expansion in $\hat{C}$ [Eqs. (A8) and (A9)]; green lines: results from Ref. [2]. 

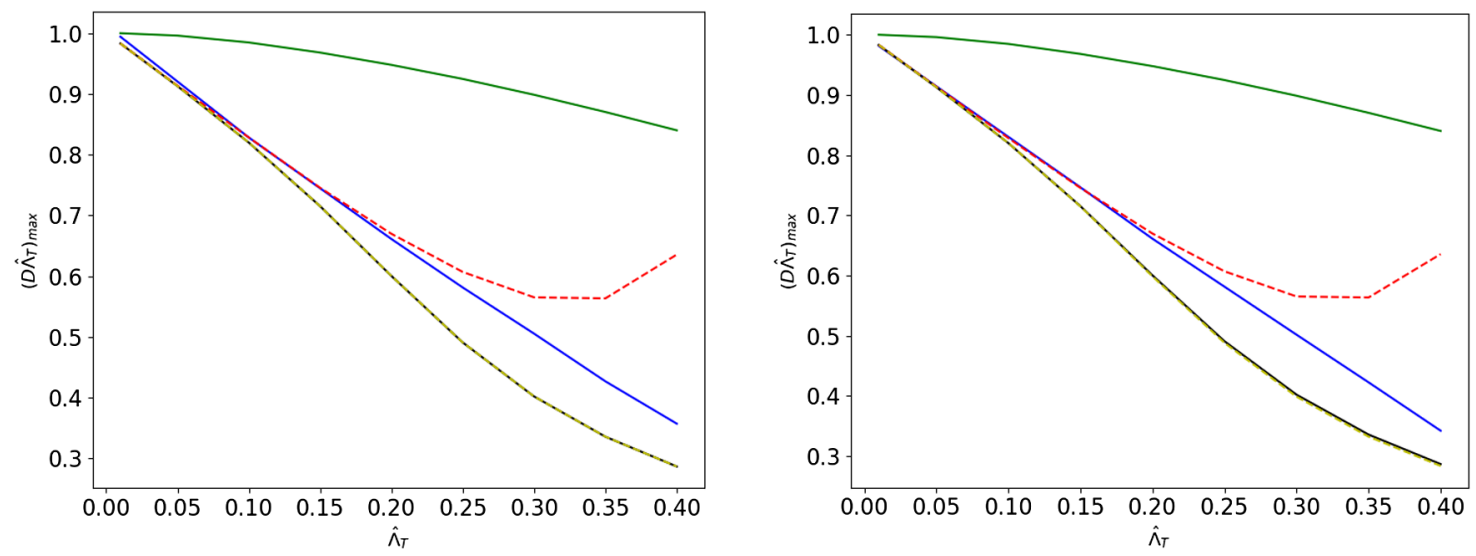

FIG. 5. Left plot: value of $D \hat{\Lambda}_{T}$ for the maxima of $G_{1}+G_{2}$ in Fig. 3, as a function of $\hat{\Lambda}_{T}$ for the case $\sigma_{\hat{C}}=0.1$. Right plot: the same for the maxima of $G_{1}+G_{2}$ in Fig. 4 , that is for $\sigma_{\hat{C}}=0.5$. Blue lines: numerical integration; black lines: zeroth effective order in $\hat{\Lambda}_{T} \ll 1$, using a zeroth order expansion in $\hat{C}$ [Eqs. (35) and (36)]; yellow dashed lines: zeroth effective order in $\hat{\Lambda}_{T} \ll 1$, using a second order expansion in $\hat{C}$ [Eqs. (A3) and (A4)]; red dashed lines: second effective order in $\hat{\Lambda}_{T} \ll 1$, using a zeroth order expansion in $\hat{C}$ [Eqs. (A8) and (A9)]; green lines: results from Ref. [2]. Only the analytical expression in Eq. (A4) depends, slightly, on $\sigma_{\hat{C}}$; the blue lines (numerical integration) vary as well. Differences are very slight, although they can be seen by inspection.

$\hat{\Lambda}_{T} \ll 1$, using a zeroth order expansion in $\hat{C}$ [Eqs. (A8)(A10)]. Finally, the green lines are the results from Ref. [2]. For the numerical case (blue line) $G_{1}+G_{2}$ can be extracted by convolving the results for $G$ with a flattop function, thus effectively averaging over the fast oscillations due to $G_{3}$.

As expected, the reader can see by inspection a better agreement between analytical results (black, yellow and red lines) and numerical results in the case for smaller values of $\hat{\Lambda}_{T}$. The expansion to the second order in $\hat{C}$ (yellow line) differs from the black line for the larger value of $\sigma_{\hat{C}}=0.5$, but differences are relatively small. All our plots, however, underline an important difference with the results of [2] (rendered with green lines). Both the expression in [2] and

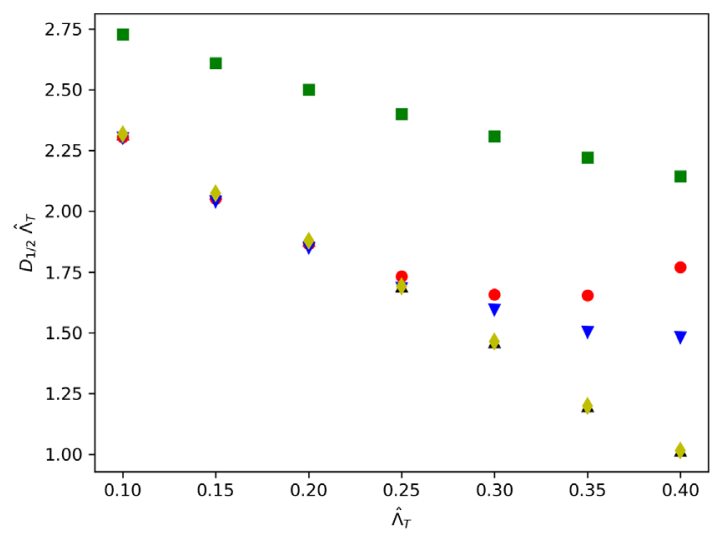

our expressions include the fast oscillations in $G_{3}$, and both are expansions for small values of $\hat{\Lambda}_{T}$ : in fact, in the limit $\hat{\Lambda}_{T} \rightarrow 0$ and $\sigma_{\hat{C}} \rightarrow 0$ all lines collapse to the same function, as it should be. The difference is not due to the order of the expansion in $\hat{\Lambda}_{T}$ or in $\hat{C}$. In fact, the effect of different expansion order can be seen by comparing the blue line (numerical calculations) with the black, yellow, and red lines (accounting for different accuracies in the order of the expansion, as defined above) and is not too large. The difference with the green curve [2] is due to the fact that in [2], terms of order $D^{n} \hat{\Lambda}_{T}^{n}$ are neglected, and in that reference the value $D \hat{\Lambda}_{T}$ which maximizes the gain is erroneously predicted to be close to unity. Figure 5 gives a

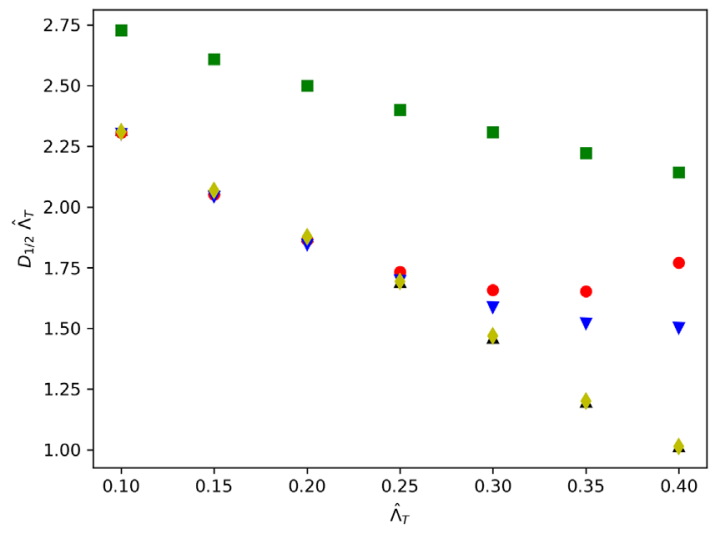

FIG. 6. Left plot: values of $D_{1 / 2} \hat{\Lambda}_{T}$ for $G_{1}+G_{2}$ (see text for further explanations) in Fig. 3, as a function of $\hat{\Lambda}_{T}$ for the case $\sigma_{\hat{C}}=0.1$. Right plot: the same for the values of $D_{1 / 2} \hat{\Lambda}_{T}$ for $G_{1}+G_{2}$ in Fig. 4, that is for $\sigma_{\hat{C}}=0.5$. Blue markers: numerical integration; black markers: zeroth effective order in $\hat{\Lambda}_{T} \ll 1$, using a zeroth order expansion in $\hat{C}$ [Eqs. (35)-(37)]; yellow markers: zeroth effective order in $\hat{\Lambda}_{T} \ll 1$, using a second order expansion in $\hat{C}$ [Eqs. (A3)-(A5)]; red markers: second effective order in $\hat{\Lambda}_{T} \ll 1$, using a zeroth order expansion in $\hat{C}$ [Eqs. (A8)-(A10)]; green markers: results from Ref. [2]. Only the analytical expression in Eq. (A4) depends, slightly, on $\sigma_{\hat{C}}$; the blue markers (numerical integration) vary as well. Differences are very slight. 
direct representation of this statement. Note that for a fixed value of $\hat{\Lambda}_{T}$, the magnitude of terms in $D^{n} \hat{\Lambda}_{T}^{n}$ depend on the value of the dispersion $D$. In the limit for vanishing small $\hat{\Lambda}_{T}$, when $D^{n} \hat{\Lambda}_{T}^{n}$ becomes of order unity, the dominant term in the expression for $G$ is of order $D^{2} \sim \hat{\Lambda}_{T}^{-2}$ (in $G_{2}$ ) and the correction is small: this leads to the collapse of all curves in the plots. However, for small, but not vanishing-small values of $\hat{\Lambda}_{T}$, the error, i.e., the difference between green and black lines, can become large both in position and amplitude. As we will see, this has an impact on energy spread estimations that cannot be neglected.

As we will see in the next section, from the previous plots one can extract information allowing to diagnose energy spread of the electron beam and coherence time of the radiation pulse. The idea of estimating the energy spread by measuring the gain as a function of the dispersion is well known, see e.g., a recent measurement in [12]. Different options are possible, and we will consider, in particular, two of them. In view of these applications we extract the position $D \hat{\Lambda}_{T}$ for which one obtains the maximum value of $G_{1}+G_{2}$, plotted against $\hat{\Lambda}_{T}$, see Fig. 5 and the value $D_{1 / 2} \hat{\Lambda}_{T}>0$, for which $\mathrm{G}$ drops to half of the value it has at $D=0$, see Fig. 6 . We do that comparing numerical calculations with various analytical approximations used in this paper.

\section{DIAGNOSTICS APPLICATIONS}

In a typical XFEL setup, like that sketched in Fig. 1, one can change the delay of the chicane in steps, and perform a phase-shifter scan for each delay step. The measured quantities for each phase-shifter scan are the mean pulse energy and the oscillation amplitude (scan results are fitted with a sinusoidal function). From the dependency of these two quantities on the delay, one can extract the uncorrelated energy spread in the electron beam as well as the coherence time (or, equivalently, the intrinsic bandwidth) of the SASE FEL radiation by comparison with the theoretical results presented in this paper.

\section{A. Energy spread diagnostics}

One can extract information on the energy spread by looking at the behavior of the function $\bar{G} \equiv G_{1}+G_{2}$. One can consider several algorithms. For example, one can fit the scan result with one of the analytical solutions presented in this paper. As one can see from Figs. 3 and 4, these solutions are sufficiently accurate up to $\hat{\Lambda}_{T} \simeq 0.1-0.2$.

Alternatively, one can use simplified algorithms for quick estimates using Figs. 7-9. These estimates can be used in a wider range of values of $\hat{\Lambda}_{T}$. The plots refer to the case $\sigma_{\hat{C}}=0.5$, though differences with smaller values are small.

At the first step one can estimate the value of $\hat{\Lambda}_{T}$ from Fig. 7, where the value $\bar{G}_{\max } / \bar{G}(0)$ is presented, by taking the correspondent maximum value of the experimental curve. For convenience, on the same figure we also present (blue dotted lines) the fit function

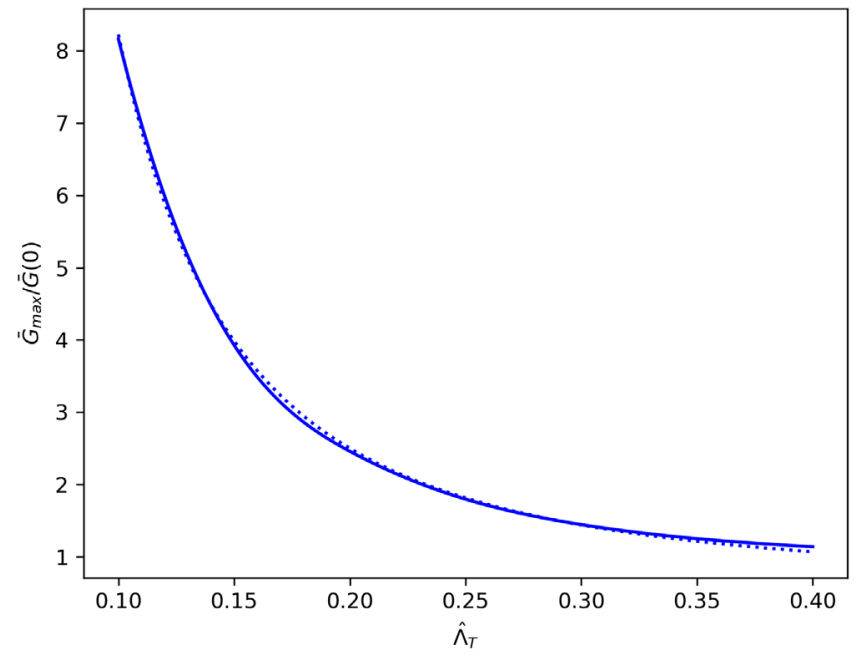

FIG. 7. Value of $\bar{G}_{\max } / \bar{G}(0)$ at $\sigma_{\hat{C}}=0.5$ (solid line). The dotted line is obtained fitting Eq. (40) to the numerical calculations.

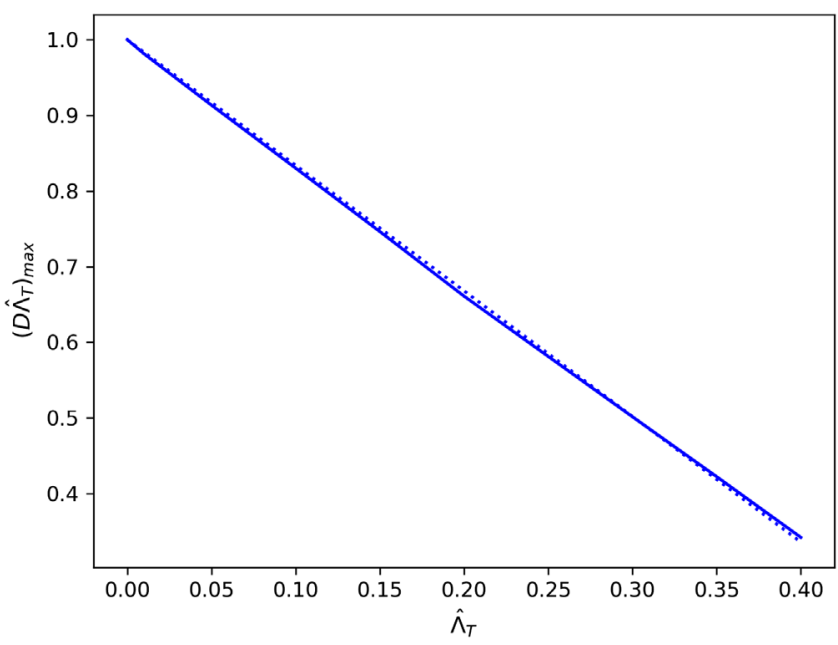

FIG. 8. Value of $D \hat{\Lambda}_{T}$ for which one obtains the maximum value of $G_{1}+G_{2}$, plotted against $\hat{\Lambda}_{T}$ for $\sigma_{C}=0.5$ (solid line). The dotted line is obtained fitting Eq. (41) to the numerical calculations.

$$
\bar{G}_{\max } / \bar{G}(0) \simeq 0.593+\frac{0.0763}{\hat{\Lambda}_{T}^{2}}
$$

to the data. Since the $\rho$ parameter is not known a priori, one needs additional information to extract an uncorrelated energy spread. It is possible, for example, to extract the position $D \hat{\Lambda}_{T}$ for which one obtains the maximum value of $G_{1}+G_{2}$, and plot it against $\hat{\Lambda}_{T}$. This is done in Fig. 8, where the dotted line shows the fit with ${ }^{7}$

\footnotetext{
${ }^{7}$ It is interesting to note that the best fit coefficient here is close to $\sqrt{3}$ that can be obtained analytically from Eq. (36) when only the terms $D$ and $D^{2}$ are kept in the brackets.
} 


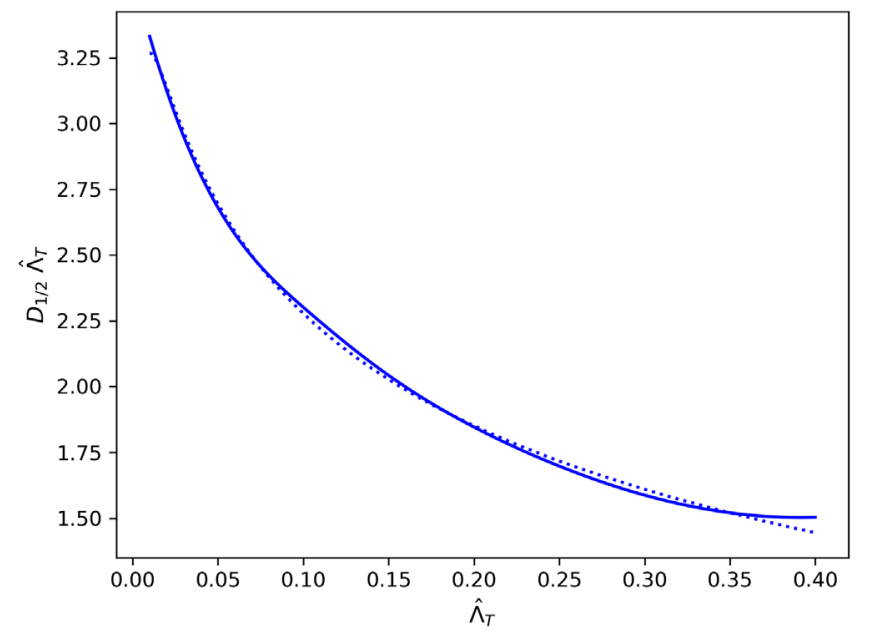

FIG. 9. Value of $D_{1 / 2} \hat{\Lambda}_{T}>0$, for which $\bar{G}$ drops to half of the value it has at $D=0$ for $\sigma_{C}=0.5$ (solid line). The dotted line is obtained fitting Eq. (42) to the numerical calculations.

$$
\left(D \hat{\Lambda}_{T}\right)_{\max } \simeq 1-1.66 \hat{\Lambda}_{T}
$$

Now one can use the previously obtained first guess for $\hat{\Lambda}_{T}$ and find $D \hat{\Lambda}_{T}=\sigma_{\delta} R_{56} \omega / c$ at the maximum. Then, from the experimentally determined $R_{56}$ at the maximum, we find the uncorrelated energy spread $\sigma_{\delta}$.

An alternative method is based on the determination of the value $D_{1 / 2} \hat{\Lambda}_{T}>0$, for which $\bar{G}$ drops to half of the value it has at $D=0$. The results of these plots are shown in Fig. 9. One can also use a fitting formula

$$
D_{1 / 2} \hat{\Lambda}_{T} \simeq-0.915-\frac{0.846}{\hat{\Lambda}_{T}^{1 / 2}}+\frac{2.72}{\hat{\Lambda}_{T}^{1 / 3}} .
$$

Once the estimates of $\hat{\Lambda}_{T}$ and $\sigma_{\delta}$ are obtained, one can also get an estimate for $\rho$.

\section{B. Coherence time diagnostics}

Analytical calculations can also be used to extract information on the coherence time, through the study of $G_{3}$. One can explicitly use the expressions for $G_{3}$ derived in the previous sections in the case for $\hat{\Lambda}_{T} \ll 1$, or can extract $G_{3}$ from the numerical calculations by subtraction of $G_{1}+$ $G_{2}$ (which is, in turn, obtained by averaging over fast oscillations as discussed before). The mathematical structure of $G_{3}$ is always $G_{3}=A \cos (\psi)+B \sin (\psi)=$ $\tilde{G} \cos (\Phi)$, with $\tilde{G}>0$. In the numerical calculations one can find $\left|G_{3}\right|=\tilde{G} \sqrt{\cos ^{2}(\Phi)}$ and then average over fast oscillations to obtain $\tilde{G}$. In the analytical case, $A$ and $B$ are known, and setting $\xi=\arctan (A / B)-\pi$ one can explicitly calculate $\tilde{G}=A \sin (\xi)+B \cos (\xi)$. The results are shown in Fig. 10 (right column) for $\sigma_{\hat{C}}=0.1$ and Fig. 11 (right column) for $\sigma_{\hat{C}}=0.5$. For the smaller $\sigma_{\hat{C}}=0.1$ we report a remarkably good agreement between the numerical calculations for $\tilde{G}$, blue lines, and the analytical case to the second effective order in $\hat{\Lambda}_{T}$ and zeroth order in $\hat{C}$, red lines. For the smaller $\sigma_{\hat{C}}=0.5$, the analytical case to the zeroth effective order in $\hat{\Lambda}_{T}$ and second order in $\hat{C}$, yellow line, is the best fit instead.

Since the dependence of $\tilde{G}$ on $\hat{\Lambda}_{T}$ and $\sigma_{\hat{C}}$ is known, once $\hat{\Lambda}_{T}$ is estimated, also $\sigma_{\hat{C}}$ can be retrieved. Since $\rho$ is already found during energy spread measurements, one can also find intrinsic FEL bandwidth. The relation with coherence time can be found in [1].

Note that a similar approach for the determination of the coherence time was developed in [11]. The difference is that in [11], the second part of the undulator is short and operates as a radiator. The theoretical results of that paper, however, consider the effect of a chicane as a delay between the electron bunch and the field only, thus ignoring any modification of the distribution function in the chicane. We think that this modification cannot be ignored. The $R_{56}$ values needed for coherent time measurements in the experiment [11] are in the order of the coherence length, and therefore much smaller than what is needed to reach the maximum of the optical klystron gain. However, these $R_{56}$ levels, albeit small, are still much larger than a wavelength, and our view is that they cannot be considered as simple delays (at variance with the case of small phase-shifter changes of the order of several wavelengths). We justify our criticism by inspecting Eq. (9), which quantifies the phase change for a finite dispersion $D$. The term $-\phi$ in Eq. (9) is actually modeling a simple delay: in fact, it does not modify the electron distribution function, because being independent of $\hat{P}$, it can be taken out of the sign of integral in the numerator of Eq. (13). Equation (13) is the expression for the field for finite dispersion $D$, and the multiplicative factor $\exp (i \phi)$ simply amounts to a delay. However, the term $D \hat{P}$ in Eq. (9) depends on $\hat{P}$ : it modifies the electron distribution function and therefore the field integral in the numerator of Eq. (13). When the phase $D \hat{P}$ is much smaller than unity, it can be neglected. The effective range of $\hat{P}$ where the integrand in the numerator of Eq. (13) is contributing to the integral is limited to values of $F^{\prime}(\hat{P})$ sensibly different from zero. By definition of the normalized momentum deviation $\hat{P}$, this is for values of $\hat{P}$ not too much larger than unity. Then, the phase $D \hat{P}$ becomes much smaller than unity and can be neglected only for values $D=\left(\rho R_{56} / \chi\right) \ll$ 1. Since $\chi / \rho$ is of order of the coherence length, the previous condition is satisfied for $R_{56}$ much smaller than the coherence length. In other words, it is fine to neglect $D \hat{P}$ (and therefore the changes to the electron distribution function) for a small phase shifter, i.e., when we discuss about $R_{56}$ values of the order of several wavelengths, but not in the case of $R_{56}$ values of order of the coherence length, which is indeed the case discussed in [11]. Similarly, one can see from $G_{3}$ that in order to measure coherence time, one needs to get to values of $D$ at least of order unity (since $\sigma_{\hat{C}} \simeq 0.5$, and in 

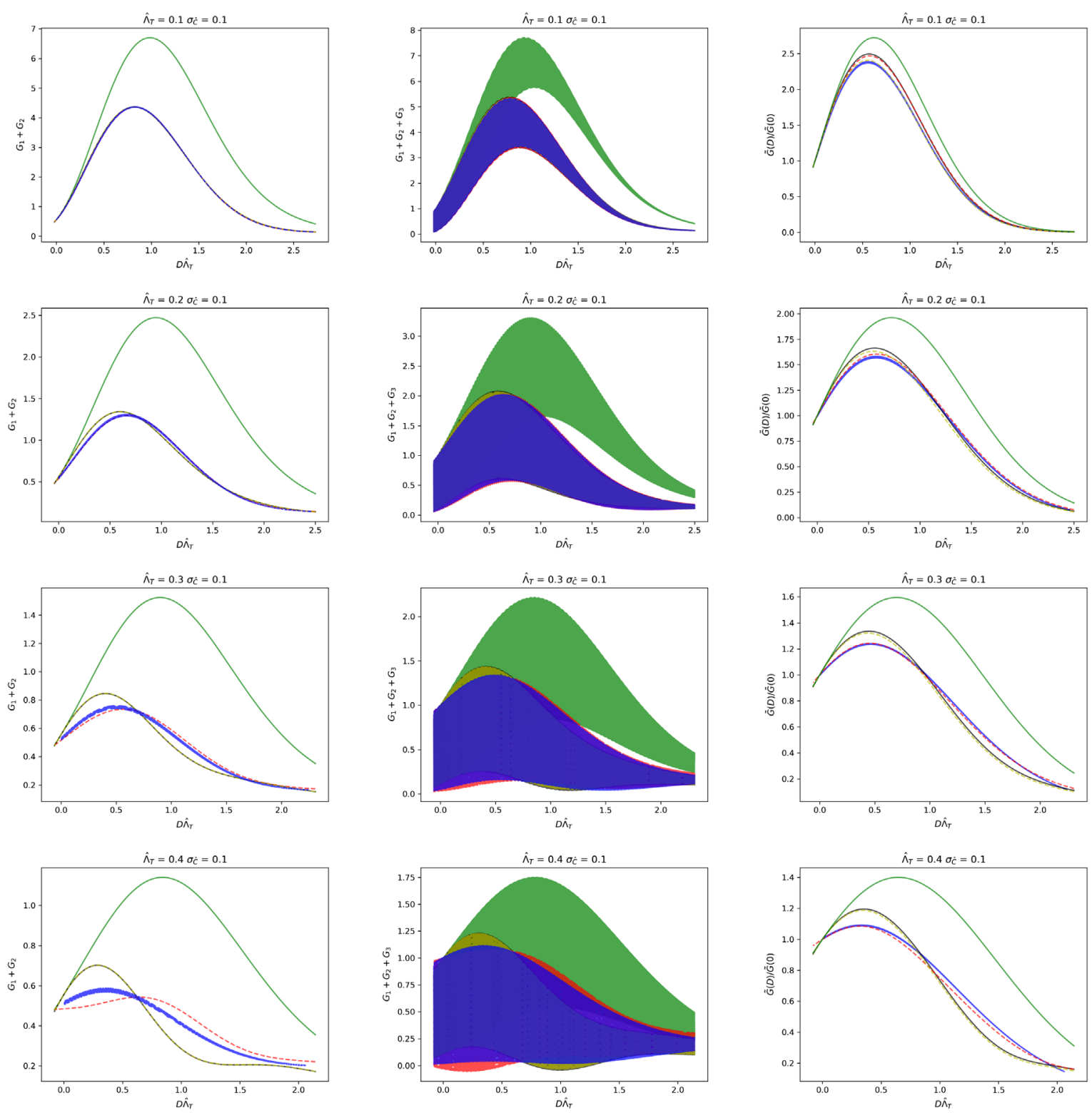

FIG. 10. $\sigma_{\hat{C}}=0.1$. Left column: comparison of $G_{1}+G_{2}$ at different values of $\hat{\Lambda}_{T}=0.1,0.2,0.3,0.4$ (rows one to four) using different methods discussed in this paper. Center column: comparison of $G_{1}+G_{2}+G_{3}$ at different values of $\hat{\Lambda}_{T}=0.1,0.2,0.3,0.4$ (rows one to four) using different methods. Right column: comparison of $\tilde{G}\left(D \hat{\Lambda}_{T}\right) / \tilde{G}(0)$ at different values of $\hat{\Lambda}_{T}=0.1,0.2,0.3,0.4$ (rows one to four) using different methods discussed in this paper. These different methods are rendered in the following way. Blue lines: numerical integration; black lines: zeroth effective order in $\hat{\Lambda}_{T} \ll 1$, using a zeroth order expansion in $\hat{C}$ [Eqs. (35)-(37)]; yellow lines: zeroth effective order in $\hat{\Lambda}_{T} \ll 1$, using a second order expansion in $\hat{C}$ [Eqs. (A3)-(A5)]; red dashed lines: second effective order in $\hat{\Lambda}_{T} \ll 1$, using a zeroth order expansion in $\hat{C}$ [Eqs. (A8)-(A10)]; green lines: results from Ref. [2].

any case it cannot be much larger than unity). Then from $G_{2}$ one sees that there is gain, which is only possible when the distribution function is modified. There is no small parameter that allows to neglect it.

\section{COMPARISON WITH LITERATURE AND CONCLUSIONS}

We reviewed optical klystron enhancement effects in SASE FELs within the framework of a 1D steady state theory, which is expected to be a relatively good approximation in the case of hard $\mathrm{x}$-ray facilities in optical klystron configurations where $\mathrm{U} 1$ and $\mathrm{U} 2$ lase in the linear regime, see Fig. 1. We found the same overall expressions already reported in literature $[2,8]$ for the optical klystron enhancement factor R, Eq. (13), and for the klystron power gain factor G, Eq. (15). However, we report relatively large differences with analytical limits reported in literature for $\hat{\Lambda}_{T} \ll 1$, which are most relevant for XFELs. These 

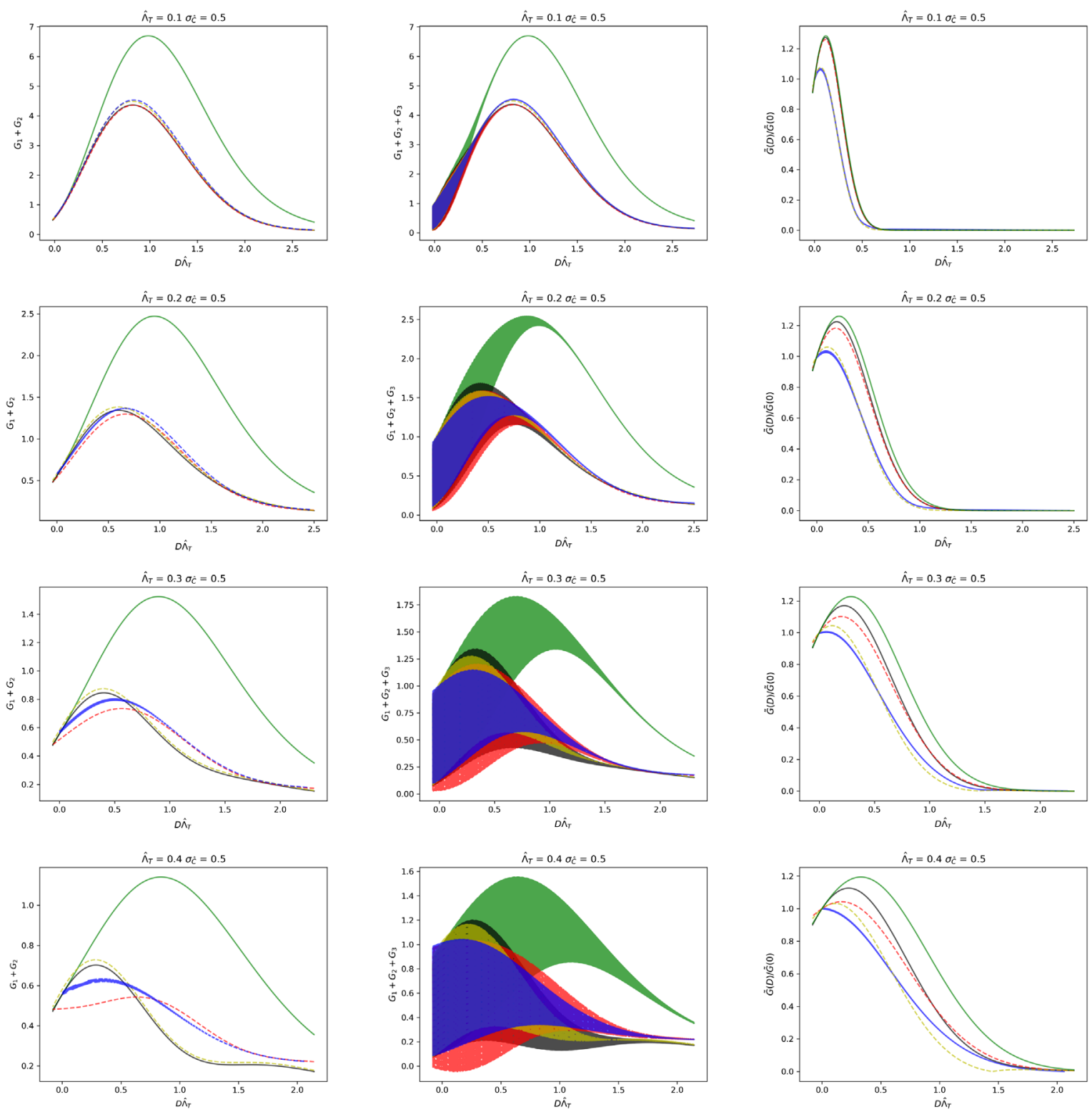

FIG. 11. $\sigma_{\hat{C}}=0.5$. Left column: comparison of $G_{1}+G_{2}$ at different values of $\hat{\Lambda}_{T}=0.1,0.2,0.3,0.4$ (rows one to four) using different methods discussed in this paper. Center column: comparison of $G_{1}+G_{2}+G_{3}$ at different values of $\hat{\Lambda}_{T}=0.1,0.2,0.3,0.4$ (rows one to four) using different methods. Right column: comparison of $\tilde{G}\left(D \hat{\Lambda}_{T}\right) / \tilde{G}(0)$ at different values of $\hat{\Lambda}_{T}=0.1,0.2,0.3,0.4$ (rows one to four) using different methods discussed in this paper. These different methods are rendered in the following way. Blue lines: numerical integration; black lines: zeroth effective order in $\hat{\Lambda}_{T} \ll 1$, using a zeroth order expansion in $\hat{C}$ [Eqs. (35)-(37)]; yellow dashed lines: zeroth effective order in $\hat{\Lambda}_{T} \ll 1$, using a second order expansion in $\hat{C}$ [Eqs. (A3)-(A5)]; red dashed lines: second effective order in $\hat{\Lambda}_{T} \ll 1$, using a zeroth order expansion in $\hat{C}$ [Eqs. (A8)-(A10)]; green lines: results from Ref. [2].

differences are not related to different accuracy chosen, but rather to the neglection, in literature, of terms of order $D^{n} \hat{\Lambda}_{T}^{n}$. This can be seen by inspection by comparing the expressions in [2], Eq. (39), with our $G=G_{1}+G_{2}+G_{3}$ with $G_{1}, G_{2}$ and $G_{3}$ given by Eqs. (35)-(37): both of these expressions for $G$ are calculated up to the zeroth order in $\hat{\Lambda}_{T}$ and both of these expressions include the fast oscillatory term $G_{3}$. However, our expressions include terms of order $D^{n} \hat{\Lambda}_{T}^{n}$ that are neglected in [2]. Since energy spread effects become important when $D$ becomes of order $\hat{\Lambda}_{T}^{-1}$, small values of $\hat{\Lambda}_{T}$ correspond to large values of $D$, and $D^{n} \hat{\Lambda}_{T}^{n}$ can never be neglected, mathematically, if an accuracy to the zeroth order in $\hat{\Lambda}_{T}$ is selected. For a fixed value of $\hat{\Lambda}_{T}$, the magnitude of terms in $D^{n} \hat{\Lambda}_{T}^{n}$ depends on the value of the dispersion $D$. In the limit for vanishing small $\hat{\Lambda}_{T}$, when $D^{n} \hat{\Lambda}_{T}^{n}$ becomes of order unity, the dominant term in the expression for $G$ is of order $D^{2} \sim \hat{\Lambda}_{T}^{-2}$ (in $G_{2}$ ) and the correction is small. This can be seen from Fig. 4: for smaller values of $\hat{\Lambda}_{T}$ the error made by neglecting terms of order 
$D^{n} \hat{\Lambda}_{T}^{n}$ becomes smaller and smaller. However, for small, but not vanishing-small values of $\hat{\Lambda}_{T}$, the error, i.e., the difference between green and black lines, becomes important both in position and amplitude. This has a major impact on energy spread estimations. ${ }^{8}$

Such an impact can be clearly seen also from Fig. 8, which shows the deviation from unity of the maximum value of $D \hat{\Lambda}_{T}$. The importance of the deviation found depends on the energy spread parameter $\hat{\Lambda}_{T}$. The theory in $[2,8]$ always predicts a maximum gain near $D \hat{\Lambda}_{T}=1$ and overestimates the actual value of the energy spread. However, the error made can be negligible or not, depending on the case under study.

To illustrate this point consider, for instance, the experimental case in [12]. For the parameters in that work and for e.g., the second case in Table II, we find $\hat{\Lambda}_{T} \simeq 0.06$, and the actual maximum at $D \hat{\Lambda}_{T} \simeq 0.9$, as can also be readily estimated from Fig. 8, so that the values for the relative energy spread are overestimated of a small amount of about 10\%. An investigator studying an FEL like FERMI, operating in the vacuum ultraviolet to extreme ultraviolet and soft x-ray range of the spectrum with very small energy spread parameters, will only make a small error. In this case, our correction is likely to be only of academic interest.

However, consider a hard x-ray FEL working with peak currents of the order of several thousand amperes. In this case, the energy spread parameter will be larger. It is not unusual to reach values $\hat{\Lambda}_{T}>0.2$. This leads to much smaller maxima at $D \hat{\Lambda}_{T}<0.6$. In this case, neglecting corrections one would overestimate the energy spread of about $40 \%$ or larger. An investigator studying the European XFEL would thus make a much more important error. In this case, our correction must be accounted for.

We derived analytical expressions for $\mathrm{R}$ and $\mathrm{G}$ to different effective orders in $\hat{\Lambda}_{T}$ and expansion in $\hat{C}$, in the limit for $\hat{\Lambda}_{T} \ll 1$ and compared them with the numerical integration of $R$ and $G$. The expressions for $G$, either found by numerical or analytical means, can be used for the determination of $\hat{\Lambda}_{T}$, and hence the energy spread of the electron beam, but also for the determination of the coherence time, through the analysis of the oscillatory part of $G$.

\section{APPENDIX: HIGHER ORDER EXPANSIONS IN $\hat{C}$ AND $\hat{\Lambda}_{T}$}

In this Appendix we treat two examples of higher order expansions in $\hat{C}$ and $\hat{\Lambda}_{T}$. We report the expressions used explicitly, as they may be interesting for cross-checking of numerical codes.

\section{Zeroth effective order in $\hat{\Lambda}_{T}$ and second order expansion in $\hat{C}$}

Using the second order expansion of the integrands Eq. (14) in $\hat{P}$, i.e., keeping the zeroth effective order in $\hat{\Lambda}_{T}$ in G, we are still free to expand $\hat{C}$ up to any order. Since we deal with the high gain case, the zeroth order expansion in $\hat{C}$ is acceptable as a limiting case, but in practice, one needs to keep higher order to account for the fact that $\sigma_{\hat{C}}$ is of order unity in the SASE case. Expanding $\hat{C}$ to the second order ${ }^{9}$ around $\hat{C}_{m} \simeq 3 \hat{\Lambda}_{T}^{2}$ and keeping up to the first effective order in $\hat{\Lambda}_{T}$ we obtain

$$
f_{1}=\frac{1}{3}+\frac{1}{9}(1+i \sqrt{3}) \hat{C}+\frac{1}{27}(-1+i \sqrt{3}) \hat{C}^{2},
$$

and the resulting expressions for $G_{1}, G_{2}$ and $G_{3}$ become, after truncation ${ }^{10}$ of terms in $\hat{C}$ of order higher than the second,

$$
\begin{gathered}
G_{1}=\frac{1}{9}+\frac{2}{81} \sigma_{\hat{C}}^{2} \\
G_{2}=\frac{1}{9} \exp \left(-D^{2} \hat{\Lambda}_{T}^{2}\right)\left(4+2 \sqrt{3} D+D^{2}-13 D^{2} \hat{\Lambda}_{T}^{2}-2 \sqrt{3} D^{3} \hat{\Lambda}_{T}^{2}+7 D^{4} \hat{\Lambda}_{T}^{4}\right) \\
+\frac{1}{81} \exp \left(-D^{2} \hat{\Lambda}_{T}^{2}\right)\left\{8+D\left[2 \sqrt{3}+D+2 D(-9-\sqrt{3} D) \hat{\Lambda}_{T}^{2}-14 D^{3} \hat{\Lambda}_{T}^{2}\right]\right\} \sigma_{\hat{C}}^{2}
\end{gathered}
$$

\footnotetext{
${ }^{8} \mathrm{~A}$ separate difference with [2], which we ascribe to a misprint in [2], is discussed in footnote 6.

${ }^{9}$ As before the phase factor in $f_{2}$ remains unexpanded.

${ }^{10}$ Truncation is implemented as a further expansion in $\hat{C}$ around zero, not anymore around $\hat{C}_{m}$.
} 


$$
\begin{aligned}
G_{3}= & \frac{1}{9} \exp \left(-\frac{D^{2}\left(\hat{\Lambda}_{T}^{2}+\sigma_{\hat{C}}^{2}\right)}{2}\right)\left[\left(4+\sqrt{3} D-4 D^{2} \hat{\Lambda}_{T}^{2}\right) \cos \left(\frac{D}{2 \rho}\right)-D \sin \left(\frac{D}{2 \rho}\right)\right] \\
& +\frac{1}{81} \exp \left(-\frac{D^{2}\left(\hat{\Lambda}_{T}^{2}+\sigma_{\hat{C}}^{2}\right)}{2}\right) D \sigma_{\hat{C}}^{2}\left[\left\{-17 \sqrt{3}-D\left[6-(10-\sqrt{3} D) \sigma_{\hat{C}}^{2}+\hat{\Lambda}_{T}^{2}\left(30-18 \sqrt{3} D+10 D^{2} \sigma_{\hat{C}}^{2}\right)\right]\right\} \cos \left(\frac{D}{2 \rho}\right)\right. \\
& \left.-\left(-11+D\left\{5 D \sigma_{\hat{C}}^{2}+6 \sqrt{3}\left(-1+\sigma_{\hat{C}}^{2}\right)+6 \hat{\Lambda}_{T}^{2}\left[-\sqrt{3}+D\left(1-\sqrt{3} D \sigma_{\hat{C}}^{2}\right)\right]\right\}\right) \sin \left(\frac{D}{2 \rho}\right)\right]
\end{aligned}
$$

Note how all these expressions are actually generalizations of those for the zeroth order in $\hat{C}$. This can be seen by inspection, as $f_{1,2}, G_{1,2,3}$ are now written as the expressions found before for the zeroth order in $\hat{C}$ plus additional terms.

\section{Second effective order in $\hat{\Lambda}_{T}$ and second order expansion in $\hat{\boldsymbol{C}}$}

In order to increase the accuracy of $G$ to the second effective order in $\hat{\Lambda}_{T}$ one needs to write $f_{1}$ and $f_{2}$ (or equivalently $R$ ) including all terms of effective order $\hat{\Lambda}_{T}^{3}$, so that the square modulus includes all terms of order $\hat{\Lambda}_{T}^{2}$. According to our previous discussion, this needs an expansion of Eq. (14) to the fourth order in $\hat{P}$. Furthermore Eq. (27), to which we add the fourth order corrections $\beta_{1} \hat{\Lambda}_{T}$, should now be used as an expression for the eigenvalue solution. As before, we can expand in $\hat{C}$ around the optimum $\hat{C}_{m}$ to any order. To be consistent we take $\hat{C}_{m} \simeq 3 \hat{\Lambda}_{T}^{2}+\beta_{2} \hat{\Lambda}_{T}^{4}$. In the following, we only report the zeroth order expansion, which leads to

$$
f_{1}=\frac{1}{3}\left(1+2 \hat{\Lambda}_{T}^{2}\right)
$$

and

$$
\begin{aligned}
f_{2}= & \frac{1}{6} \exp \left(-i \hat{C} D+\frac{i D}{2 \rho}\right)\left[-D\left(\hat { \Lambda } _ { T } ^ { 2 } \left\{-D\left[-4+\hat{\Lambda}_{T}^{2}\left(-D\left\{2 \hat{\Lambda}_{T}^{2}\left[-i D\left(-5 D \hat{\Lambda}_{T}^{2}+2 i+2 \sqrt{3}\right)-29 i+3 \sqrt{3}\right]+3 i-3 \sqrt{3}\right\}\right.\right.\right.\right.\right. \\
& \left.\left.\left.+4(4-3 i \sqrt{3}))]+(-9 \sqrt{3}+29 i) \hat{\Lambda}_{T}^{2}+5(\sqrt{3}-i)\right\}-i-\sqrt{3}\right)-4 \hat{\Lambda}_{T}^{2}+4\right] .
\end{aligned}
$$

Note that $\beta_{2}$ would enter in the expression for $f_{2}$ only if we expanded the phase, which we do not. Since we keep up to the third effective order in $\hat{\Lambda}_{T}$ it becomes unimportant for the later calculation of $G$. In fact, keeping up to the second order in $\hat{\Lambda}_{T}$ we have

$$
\begin{gathered}
G_{1}=\frac{1}{9}\left(1+4 \hat{\Lambda}_{T}^{2}\right), \\
G_{2}=\frac{1}{9} \exp \left(-D^{2} \hat{\Lambda}_{T}^{2}\right)\left(4+2 \sqrt{3} D+D^{2}-13 D^{2} \hat{\Lambda}_{T}^{2}-2 \sqrt{3} D^{3} \hat{\Lambda}_{T}^{2}+7 D^{4} \hat{\Lambda}_{T}^{4}\right. \\
\left.+2 \hat{\Lambda}_{T}^{2}\left\{-4-D\left[6 \sqrt{3}-D \hat{\Lambda}_{T}^{2}\left(32-D\left\{-9 \sqrt{3}-D \hat{\Lambda}_{T}^{2}\left[-25-3 D\left(\sqrt{3}-2 D \hat{\Lambda}_{T}^{2}\right)\right]\right\}\right)\right]\right\}\right),
\end{gathered}
$$

and

$$
\begin{aligned}
G_{3}= & \frac{1}{9} \exp \left(-\frac{D^{2}\left(\hat{\Lambda}_{T}^{2}+\sigma_{\hat{C}}^{2}\right)}{2}\right)\left[\left(\left(4+\sqrt{3} D-4 D^{2} \hat{\Lambda}_{T}^{2}\right)+\hat{\Lambda}_{T}^{2}\left\{4-D\left[3 \sqrt{3}-D \hat{\Lambda}_{T}^{2}\left(8+3 \sqrt{3} D-4 D^{2} \hat{\Lambda}_{T}^{2}\right)\right]\right\}\right)\right. \\
& \left.\times \cos \left(\frac{D}{2 \rho}-3 D \hat{\Lambda}_{T}^{2}\right)+D\left[-1+\hat{\Lambda}_{T}^{2}\left(-7+3 D^{2} \hat{\Lambda}_{T}^{2}\right)+\hat{\Lambda}_{T}^{3}\left(12 \sqrt{3} D \hat{\Lambda}_{T}-4 \sqrt{3} D^{3} \hat{\Lambda}_{T}^{3}\right)\right] \sin \left(\frac{D}{2 \rho}-3 D \hat{\Lambda}_{T}^{2}\right)\right]
\end{aligned}
$$

which would only include terms of order $D \beta_{2} \hat{\Lambda}_{T}^{4}$ in the arguments of the sin and cos functions in Eq. (A10) only, but can be neglected because we keep up to the second effective order in $\hat{\Lambda}_{T}$. It can be seen by inspection that neglecting effective orders in $\hat{\Lambda}_{T}$ yields back the results in the main text as it should be. 
[1] S. Saldin, E. Schenidmiller, and M. Yurkov, The Physics of Free-Electron Lasers (Springer, New York, 2000).

[2] Y. Ding, P. Emma, Z. Huang, and V. Kumar, Phys. Rev. ST Accel. Beams 9, 070702 (2006); 23, 019901(E) (2020).

[3] N. A. Vinokurov and A. N. Skrinsky, Report No. INP 7759, Novosibirsk, 1977.

[4] R. Bonifacio, R. Corsini, and P. Pierini, Phys. Rev. A 45, 4091 (1992).

[5] N. A. Vinokurov, Nucl. Instrum. Methods Phys. Res., Sect. A 375, 264 (1996).

[6] K. J. Kim, Nucl. Instrum. Methods Phys. Res., Sect. A 407, 126 (1998).
[7] E. L. Saldin, E. A. Schneidmiller, and M. V. Yurkov, in Proceedings of the 2004 Free Electron Laser Conference, Trieste, Italy, 2004 (Comitato Conferenze Elettra, Trieste, 2005), p. 143.

[8] G. Penco, E. Allaria, G. D. Ninno, E. Ferrari, L. Giannessi, E. Roussel, and S. Spampinati, Photonics 4, 15 (2017).

[9] S. Liu et al., Phys. Rev. ST Accel. Beams 22, 060704 (2019).

[10] S. Serkez et al., Appl. Sci. 10, 2728 (2020).

[11] G. Zhou et al., Sci. Rep. 10, 5961 (2020).

[12] G. Penco, E. Allaria, G. De Ninno, E. Ferrari, and L. Giannessi, Phys. Rev. Lett. 114, 013901 (2015). 